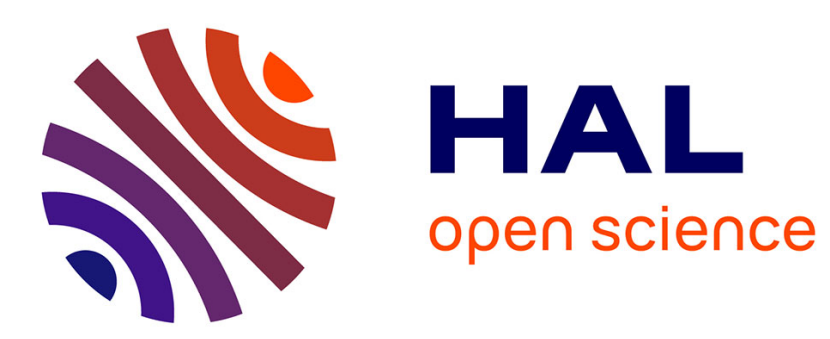

\title{
Molecular simulation of the water-triolein-oleic acid mixture: Local structure and thermodynamic properties
}

Estelle Couallier, A. Riaublanc, E. David Briand, B. Rousseau

\section{To cite this version:}

Estelle Couallier, A. Riaublanc, E. David Briand, B. Rousseau. Molecular simulation of the watertriolein-oleic acid mixture: Local structure and thermodynamic properties. Journal of Chemical Physics, 2018, 148 (18), 10.1063/1.5021753 . hal-01823874

\section{HAL Id: hal-01823874 https://hal.science/hal-01823874}

Submitted on 7 Jun 2021

HAL is a multi-disciplinary open access archive for the deposit and dissemination of scientific research documents, whether they are published or not. The documents may come from teaching and research institutions in France or abroad, or from public or private research centers.
L'archive ouverte pluridisciplinaire $\mathbf{H A L}$, est destinée au dépôt et à la diffusion de documents scientifiques de niveau recherche, publiés ou non, émanant des établissements d'enseignement et de recherche français ou étrangers, des laboratoires publics ou privés. 
PublishiMgolecular simulation of the water-triolein-oleic acid mixture: local structure and thermodynamic properties

E. Couallier, ${ }^{1, \text { a) }}$ A. Riaublanc, ${ }^{2}$ E. David Briand, ${ }^{2}$ and B. Rousseau ${ }^{3}$

1) CNRS UMR 6144, GEPEA, 37 Boulevard de l'université, BP 406, 44602 Saint-Nazaire cedex, France

2) INRA BIA, Rue de la Géraudière, BP 71627, 44316 Nantes Gedex 3, France

3) CNRS UMR 8000, LCP, Université Paris Sud, 310 Rue Michel Magat, 91400 Orsay, France

(Dated: April 2, 2018) 
An artificial oil in water emulsion analogue to those found in bioresources or food matrices is simulated and studied experimentally. It is composed of one of the major natural free fatty acids (the oleic acid, OA) and the corresponding triacylglyceride (trioleic glyceride, or triolein, GTO). Because of the large time and length scales involved, the molecular simulations are done with the Martini force field, a coarsegrained model. This allowed us to study the water-OA-GTO system at different compositions with more than 20000 molecules and up to $2 \mu \mathrm{s}$.

Interfacial tension was measured using the pendant drop method and compared with molecular simulation results. We observe very good agreement at high OA concentration and deviations up to $15 \%$ at low OA concentrations. The water solubility in the lipid phase is in fair agreement with experiments, between 0.03 and $0.32 \mathrm{~mol} / \mathrm{L}$, rising with the OA content.

The area occupied by OA and GTO at the interface between water and pure product fitted with experimental data $\left(A_{\mathrm{OA}}=36.6 \AA^{2}\right.$ and $\left.A_{\mathrm{GTO}}=152.1 \AA^{2}\right)$. The consistency between simulation and experimental results allowed a structural analysis of the interface. A bilayer structure of the lipids at the water/oil interface is proposed, containing preferentially oleic acid but also triolein. Through all these results the usefulness of coarse-grained simulation for the description of water-oil interfacial organization is demonstrated. This method will be used later on to bring local information on the organization of target compounds, necessary in biomass fractionation processes or food additives formulations for example.

Keywords: oleic acid, triolein, interfacial tension, emulsion, coarse-grained simulations

a) estelle.couallier@univ-nantes.fr 
The local organization of lipids in complex mixtures, like oil in water emulsions, is more and more needed in different application fields. Its knowledge is necessary for the development of innovative extraction and separation processes, in the context of renewable bioresources valorization (i.e. microalgae) for food, feed (polyunsaturated lipids) and energy (triglycerides) ${ }^{1}$. For example, the many different lipids produced by microorganisms are dispersed in aqueous phase after grinding and a suitable fractionation strategy must be employed. It was shown that lipids with different unsaturated chains don't mix together and form rafts at fat globule interfaces, and that triglyceride organization at the globule interface, changing with temperature, may modify the interfacial properties ${ }^{2}$. Depending on the detailed composition, the molecules can be at the lipid droplet interface, in the lipid bulk, organized as liquid crystals or oleosome in the aqueous phase etc. and the separation procedure may vary.

Thus, the determination of the local organization (3D structure with eventual heterogeneity) will allow building fractionation strategies (for example destabilization of aggregates by shear rate or temperature variation to induce reorganization). It will help to understand the impact of molecular organization at the oil-water and liquid-solid interfaces that are key parameters for the processes.

It becomes also important to understand the behavior of the polyunsaturated lipids ( $\omega-3$ and $\omega-6$ ) that have nowadays to be introduced in food matrices, because of recent nutritional recommendations. Innovative formulations containing the unsaturated fatty acids also have to prevent their oxidation or degradation. Berton et $a .^{3}$ have shown that the oxidation of fragile unsaturated lipids depends on the nature of the water-oil droplet interface composition. A controlled local structure is therefore needed. The organization of lipids in emulsions is also very important in the domain of cosmetics and medicine, for controlled drug delivery for example.

Unfortunately, direct observation of local organization through experimental means is difficult $^{2}$ and/or expensive ${ }^{4,5}$. Molecular simulations are becoming a tool of growing importance, applied to different systems, from simple fluids to complex biomolecules, soft-matter and nano-materials. By looking at phenomena at the molecular scale, computer simulations 
Publishing tribute to a better understanding of the structure-property relationships. It is a remarkable tool to investigate the effect of small changes in molecular structure or composition onto spatial organization for e.g. surfactants in water or lipid in water emulsion stability.

One requirement for reliable molecular simulations is a correct sampling of the various degrees of freedom in the system. When using atomistic molecular dynamics, a correct sampling can be achieved if simulated times exceed the largest relaxation time in the system. In a recent paper, Skjevik et al. ${ }^{6}$ have used large GPU based computers to study lipid bilayer self-assembly. They have shown that the typical timescale for the formation of a phospholipid bilayer is of the order of a few hundreds of nanoseconds, a quite large value for all-atom simulations. Therefore, the timescales involved in the physical processes studied are too high to be studied systematically by molecular simulation at the atomistic level.

One route to reach larger time and length scales is to adopt coarser models where particles represent a group of atoms, typically between 3 and 10 heavy atoms (C, N, O, S, etc). By reducing the number of particles to handle (for a given physical system), simulations can be realized over a reduced computing time. Moreover, because interaction forces between particles are softer, larger timesteps can be employed during the simulations. Finally, accessible timescales can be increased by two or more orders of magnitude, depending on the details of the coarse-graining procedure.

The second requirement for molecular simulation is a good description of the interparticle forces. These forces are described empirically by simple functions of the particle positions. The set of parameters plus the analytical force expressions is called the force field (FF). In recent years, many efforts have been made to derive coarse-grained (CG) force fields adapted to different types of molecules (see e.g. ${ }^{7}$ ). However, when dealing with lipids, the Martini FF appears to be one of the most used and validated ${ }^{8}$. As every coarse-grained force field, dynamics is usually faster than real one, although a global time scaling factor seems to apply when using the Martini force field. As mentioned by Baron et al. ${ }^{9}$, thermodynamic properties could be reproduced provided the CG model is re-parameterized on the basis of the some liquid-phase properties. Recently, Bereau and Kremer ${ }^{10}$ proposed an automated parameterization procedure for common small organic molecules. The method was able to reproduce the water-octanol partitioning free energy of more than 650 neutral compounds with a deviation of the order of magnitude of the thermal energy at room temperature. One of the advantages of the original Martini force field is its limited set of parameters, 
Publishiwgich make it relatively easy to adjust or optimize the interactions ${ }^{11}$. Although it was designed primarily for lipids (free fatty acids, triglycerides, phospholipids, cholesterol etc. ${ }^{12,13}$ ) extensions to proteins have been proposed ${ }^{14}$ as well as carbohydrates ${ }^{15}$.

Recently, Neyt et al. ${ }^{16}$ compared atomistic and coarse-grained (Martini force field) predictions for the interfacial tension (IT) at the oil-water interface. They mention that CG models are able to reproduce qualitatively the effect of the salinity on the interfacial tension of the system, although a more developed calibration should be necessary for more quantitative results. Ndao et al. ${ }^{17}$ applied the same methodology to the prediction of interfacial tension in several organic molecule-water systems. They also insist on the need for a re-parameterization to better match the interfacial tension. They emphasize the fact that liquid-liquid interface equilibration is an issue which can be partially resolved using CG rather than all-atoms models.

In this work, an artificial oil in water emulsion analogous to those found in bioresources or food matrices is simulated, composed of a limited number of compounds: oleic acid and the corresponding triacylglyceride, trioleic glyceride, or triolein. Before studying oil mixtures in water, force field parameter for the OA head is calibrated against the water-OA interfacial tension. Then we investigate molecyle organization, mainly at the interface, and its impact on a macroscopic parameter, the interfacial tension of the mixture. Results are compared with our experiments and results from literature (interfacial tension, water solubility in oil, area per molecule). We demonstrate the relevance of the CG simulation to describe the water-oil interfacial behavior of organic compounds.

\section{METHODOLOGY}

\section{A. Experiments}

Experiments were performed to measure the interfacial tension. The oily mixtures were composed of pure glyceryl trioleate (purity grade $\geq 99 \%, 885.43 \mathrm{~g} \mathrm{~mol}^{-1}$, Sigma-Aldrich) and oleic acid (purity grade $\geq 99 \%, 282.46 \mathrm{~g} \mathrm{~mol}^{-1}$, Sigma-Aldrich). The proportion of each compound was adjusted with an automatic pipette and verified through weighting. The oleic acid proportion in the organic phase was $0,5,10,20,40,60,80,100 \%_{v / v}$. The density of glyceryl trioleate, oleic acid and water are respectively $0.910 \mathrm{~kg} \mathrm{~L}^{-1}, 0.890 \mathrm{~kg} \mathrm{~L}^{-1}$ 
Publishi(frgema-Aldrich data) and $0.99786 \mathrm{~kg} \mathrm{~L}^{-1}$. The density of a mixture GTO-OA $(40 / 60 \mathrm{v} / \mathrm{v})$ was measured at $0.904 \mathrm{~kg} \mathrm{~L}^{-1}$.

The interfacial tension between oil and water was measured using the pendant drop method (Drop Shape Analyser DSA 30, Krüss) at a constant temperature of 295 K. A pendant water drop was formed with a clean disposable syringe filled with ultrapure water associated to a needle (diameter $1.8 \mathrm{~mm}$ ) inside a $8 \mathrm{~mL}$ cuve made of quartz filled with lipid phase, so that the drop profile allow a mathematical analysis with a model based on the Young-Laplace equation coupled with the calculation of the pressure difference between inside and outside the drop ${ }^{18,19}$ (equation 1). The shape factor defined by Song ${ }^{19}$ was $>0.5$ to minimize the interfacial tension error. The experimental densities of oil and water were input parameters and were supposed constant. Equilibrium was reached after a few minutes. Finally, the interfacial tension was obtained from the equation 1 . The schematic droplet profile is presented in figure 1.

$$
\gamma\left(\frac{1}{R_{1}}+\frac{1}{R_{2}}\right)=\frac{2 \gamma}{R_{0}}+\Delta \rho g z
$$

with $\gamma$, the interfacial tension, $R_{0}, R_{1}$ and $R_{2}$, curvature radii of the non-spherical interface, $\Delta \rho$, the density difference between water and oil, $g$, the gravitational acceleration, $z$, the vertical height measured from the datum plane.

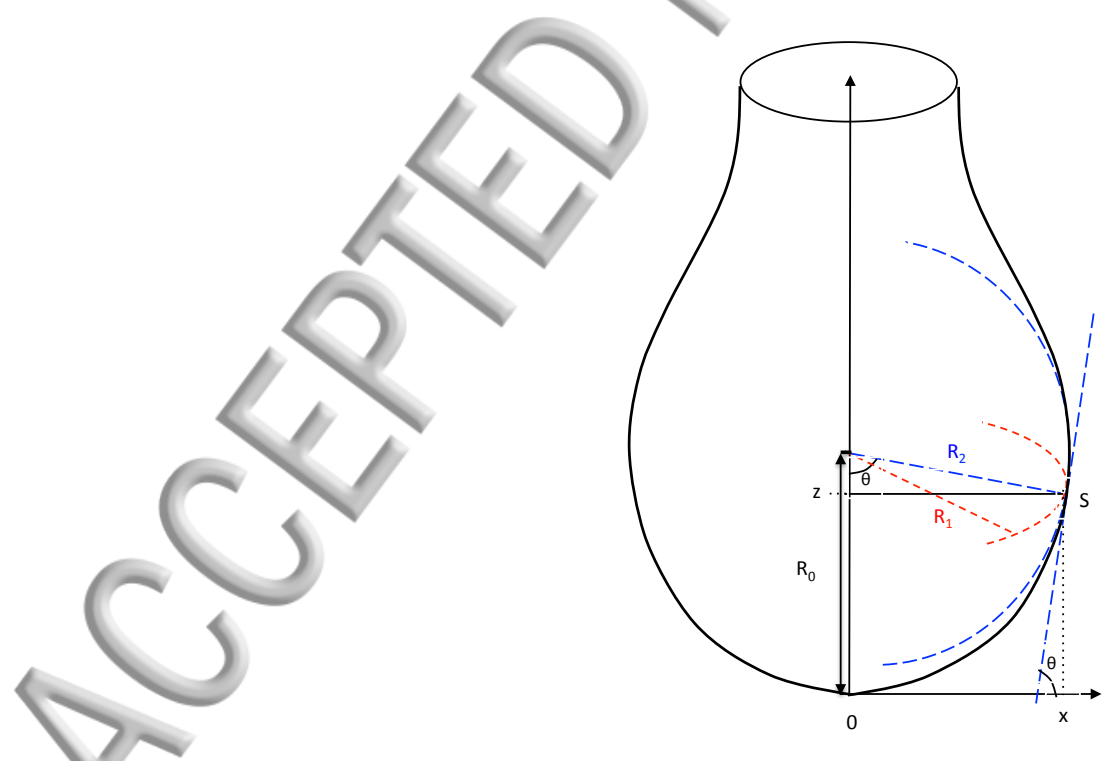

Figure 1: Pendent drop with curvature radii $R_{0}, R_{1}$ and $R_{2}$ at an arbitrary point $S$ on a convex surface 
This manuscript was accepted by J. Chem. Phys. Click here to see the version of record.

\section{Molecular Coarse Grained Simulations}

We used the MARTINI force field ${ }^{13}$ to describe interactions between all particles in the system. The Martini force field has been optimized to reproduce partitioning free energy of a large number of chemicals between polar and non-polar phases. Each particle represents a small number of atoms, typically 3 or 4 heavy atoms. The force field considers only four main types of interaction sites and up to 18 subtypes. Hence, the use of MARTINI force field first requires to represent the molecules of interest into coarse-grained particles and to associate them with the correct subtype. The force field parameters are given in the supplementary information.

\section{Modeled molecules}

In this work, four water molecules are modeled as a single $\mathrm{P}_{4}$ particle. In order to prevent the coarse-grained water model fromfreezing, so called "antifreeze" water particles have been introduced at a mole fraction of 0.1 in water ${ }^{13}$. Anti-freeze particles are of subtype $\mathrm{BP}_{4}$ with identical parameters as $\mathrm{P}_{4}$ particles. The antifreeze effect is obtained by scaling up the Lennard-Jones parameters between $\mathrm{BP}_{4}$ and $\mathrm{P}_{4}$ particles. The Lennard-Jones size parameter $\sigma$ is changed to $0.57 \mathrm{~nm}$ and the interaction energy parameter $\varepsilon$ is equal to $5.6 \mathrm{~kJ} \mathrm{~mol}^{-1}$.

The cis-9-Octadecenoic acid (OA) is composed of a carboxylic head and a long hydrophobic tail with one unsaturated bond. At the MARTINI coarse-grained level, it is composed of three particle types and a total of five particles, as shown in figure 2a. The OA tail is modeled with three $\mathrm{C}_{1}$ beads and one slightly more polar bead of type $\mathrm{C}_{3}$ to account for the unsaturation. We considered that the acid head $-\mathrm{CH}_{2} \mathrm{COOH}$ is neutral of main type $\mathrm{P}$. From the paper by Marrink et al. ${ }^{13}$, the most natural choice would be the type $\mathrm{P}_{3}$ which corresponds to acetic acid. However, other choices are possible. In a study of $\mathrm{pH}$ effect on

oleic acid aggregates, Bennett et al. ${ }^{20,21}$ used parameters adapted from aspartic acid from the MARTINI 2.1 force field ${ }^{13}$. The amino-acid region is modeled as a $\mathrm{P}_{3}$ bead in aspartic acid whereas it is described as $\mathrm{P}_{1}$ in serine, threonine, lysine and glutamic acid. We found a model for palmitic acid where the head group is represented by a $\mathrm{P}_{4}$ bead ${ }^{22}$. Therefore, as will be shown below, we decided to select the correct subtype by comparison between the experimental and the simulated interfacial tension of the pure OA with water interface. 
Publishifigery $\mathrm{P}$ subtype was studied, but $\mathrm{P}_{5}$ which is more polar than water. OA molecule will then be described as: $\mathrm{P}_{i}-\mathrm{C}_{1}-\mathrm{C}_{3}-\mathrm{C}_{1}-\mathrm{C}_{1}$.

Glyceryl trioleate parameters are taken as provided by Timu Vuorela on the MARTINI web $\operatorname{site}^{22}$. This parameter set is based on standard MARTINI 2.1 force field plus angle potential refinement as described in Vuorela et al. ${ }^{23}$. A schematic of the GTO molecule along with the coarse-grained types is given in figure 2b. Each bead represents four heavy atoms, but the glyceryl bead, composed of three heavy atoms only. Each tail is described by a non-polar group of subtype $\mathrm{N}_{\mathrm{a}}$ corresponding to the ester, followed by an OA tail $\left(-\mathrm{C}_{1}-\mathrm{C}_{3}-\mathrm{C}_{1}-\mathrm{C}_{1}\right)$ leading to a general coarse-grained structure: $\mathrm{C}_{1}\left(-\mathrm{Na}_{-}-\mathrm{C}_{1}-\mathrm{C}_{3}-\mathrm{C}_{1}-\mathrm{C}_{1}\right)_{3}$.

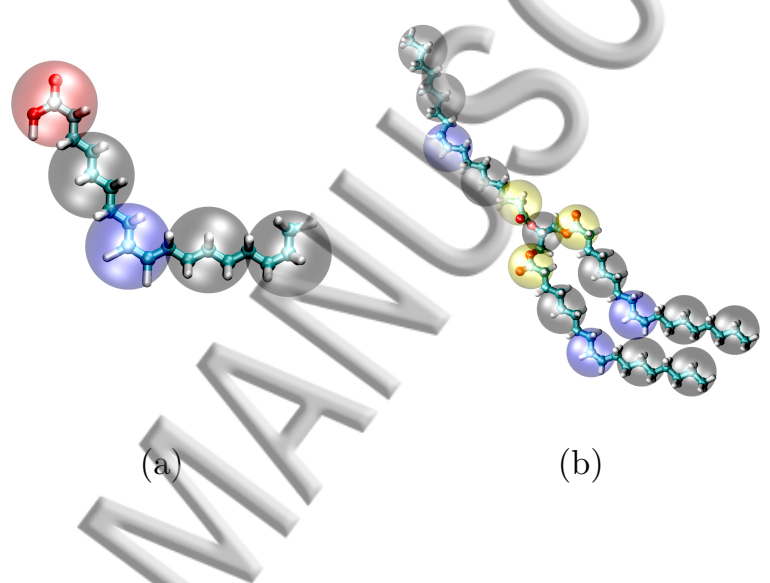

Figure 2: Schematic of (a) OA and (b) GTO molecules at both atomistic and coarse-grained description levels. Each coarse-grained particle contains four heavy atoms. Particle types are $\mathrm{P}$ (red), $\mathrm{N}_{\mathrm{a}}$ (yellow), $\mathrm{C}_{1}$ (black) and $\mathrm{C}_{3}$ (blue).

\section{Simulation tools}

Molecular dynamics simulations were done with the LAMMPS $\operatorname{code}^{24}$. We controlled LAMMPS input parameters in order to reproduce precisely the interactions that were used during force field optimisation. Thus, dispersion-repulsion interactions are described using the Lennard-Jones 12-6 potential with energy and forces vanishing at a cut off distance $r_{c}$. This is implemented in LAMMPS using a standard shift function (third-order polynomial) $)^{9}$. The Lennard-Jones potential was shifted between $r_{\text {shift }}=0.9 \mathrm{~nm}$ and $r_{\mathrm{c}}=1.2 \mathrm{~nm}$. Lennard-Jones interactions also apply between second (and more) nearest neighbors inside each molecule. Bonded interactions between adjacent beads are described by an harmonic 
Publishijpgtential of the corresponding bead distance and chain bending is represented using an harmonic potential of the cosine of angles between adjacent bonds. Equations of motion are integrated using the velocity-Verlet algorithm as implemented in LAMMPS with a time step of 20 fs. During $N V T$ simulations and $N P_{z z} T$ simulations (see below), a time constant of $40 \mathrm{ps}$ is used for the thermostat and a time constant of $400 \mathrm{ps}$ is used for the barostat.

\section{Simulation}

In this work, the influence of the oil phase composition onto the oil-water interfacial tension, $\gamma$, is studied. For all studied systems, the water amount is constant and roughly represents one third of the system volume. Nine-different systems were simulated with different OA to GTO ratio. Detailed compositions are giyen in table I. The initial OA molar fraction was calculated according to equation 2 using the initial amount of OA and GTO molecules in the simulation box, $n_{\mathrm{OA}}^{0}$ and $n_{\mathrm{GTO}}^{0}$, respectively.

$$
x_{\mathrm{OA}}^{0} \neq \frac{n_{\mathrm{OA}}^{0}}{n_{\mathrm{OA}}^{0}+n_{\mathrm{GTO}}^{0}}
$$

Initial configurations were built using the PACKMOL package ${ }^{25}$ to speed up the equilibration part. The shape of the simulation box is parallepipedic. Box length in $z$ direction is four times the value in $x$ and $y$ directions. Two interfaces are created normal to the $z$ direction and water molecules are initially separated from oil molecules. OA and GTO molecules are randomly distributed inside the oil phase. After creation, the total energy of the box is minimized using a conjugate gradient method. Then, box volume is rescaled in order to bring the system close to the experimental density. The system is then allowed to relax for $160 \mathrm{~ns}$ at $300 \mathrm{~K}$ and 1 bar using the isothermal-isobaric algorithm of Shinoda et $a l{ }^{26}$. More precisely, the pressure along the $z$ axis, $P_{z z}$, is imposed during the run and box length in $z$ direction is allowed to change while box lengths in $x$ and $y$ directions remain constant. This step enables to reach mechanical equilibrium, a crucial step before computing interfacial tension. During this $N P_{z z} T$ run, the average box length in the $z$ direction is computed over the last $100 \mathrm{~ns}$ where a plateau clearly appears. This average box length is therefore applied to the simulation box. Typical box dimensions are 107 x107 x $465 \AA$. 
Publishifigially, a very long production run of $2 \mu \mathrm{s}$ is realized for each system in the $N V T$ ensemble at $300 \mathrm{~K}$ and equilibrium properties are computed along the last $1.6 \mu \mathrm{s}$.

Some verifications were made but not detailed in the paper: we checked that the demixing arises if we start with all species placed randomly; we verified on system 2 that the interfacial tension value and the final organization are identical if all OA molecules are initially placed at the oil-water interface or placed randomly into the oil phase; it was also verified that the temperature change between 290 and $310 \mathrm{~K}$ has a negligible impact on the interfacial tension.

\section{Interfacial tension}

Interfacial tension $\gamma$ can be computed from the following expression (known as the Kirkwood-Buff expression) ${ }^{27}$ :

$$
\gamma_{\mathrm{KB}}=\frac{L}{2}\left\langle P_{\perp}-P_{\|}\right\rangle
$$

where $L$ is the box length in the $z$ direction (normal to the interface) and $P_{\perp}$ and $P_{\|}$are the instantaneous pressure components, respectively normal and parallel to the interface. The factor one half comes from the fact that two interfaces appear in the simulation box. The ensemble average is taken over the canonical ensemble. During the course of the simulation, the full pressure tensor is computed, from which instantaneous values of $P_{\perp}=P_{z z}$ and $P_{\|}=1 / 2\left(P_{x x}+P_{y y}\right)$ are deduced.

It must be noted that equation 3 is valid only in the limit where the range of interaction forces is small relative to the distance $L$. In the practical case of molecular simulation, it means that the simulation box must be large enough to avoid any interaction between the two oil-water interfaces. This can be checked by looking at the pressure profil along the direction normal to the inferface. In this work, we used the algorithm proposed by Nakamura et al. ${ }^{28}$ to compute the local pressure tensor in Cartesian coordinates in LAMMPS. In this approach, the simulation box is divided into 300 slices parallel to the interface and the pressure tensor elements are computed inside each region. The slice thickness varied between $1.55 \AA$ and $1.58 \AA$ depending on the system studied. Figure 3 shows the evolution of $P_{\perp}(z)$ and $P_{\|}(z)$, local values of pressure components in a slice around $z$. We observe that $P_{\perp}(z)$ is constant along the $z$ axis, as expected for a system at mechanical equilibrium. On the contrary, large variations of $P_{\|}(z)$ are observed around layers 50 and 230, corresponding to the location 
Publishipfgthe water-oil interfaces. However, both components are identical far from interfaces, indicating that the simulation box is large enough to compute the interfacial tension. In this work, the interfacial tension will be computed using the local pressure components ${ }^{29,30}$ :

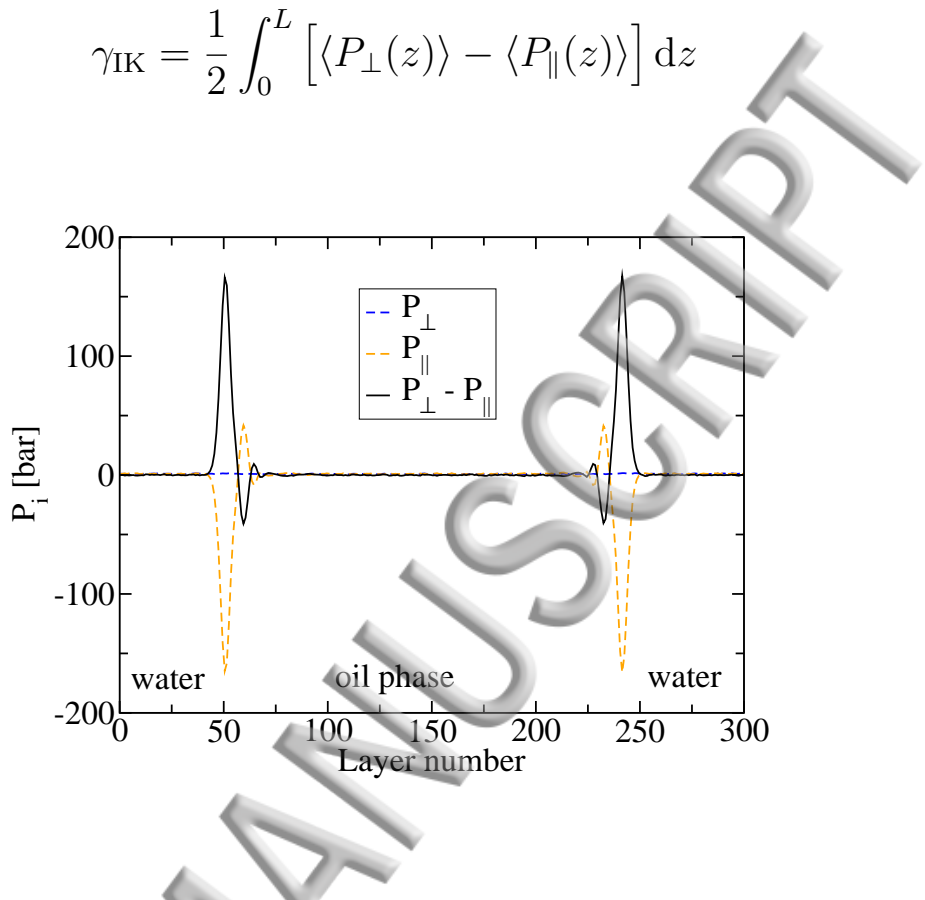

Figure 3: Local pressure components for system 7 (see table I), along the $z$ direction. The time average normal component $\left\langle P_{\perp}(z)\right\rangle$ is constant along the $z$ axis, as expected for a system at mechanical equilibrium. The location of interfaces can be clearly seen around layers 50 and 230, where large variations of the pressure component $\left\langle P_{\|}(z)\right\rangle$ occur. Layer width for system 7 is $0.156 \mathrm{~nm}$.

\section{RESULTS}

\section{A. Parameterization of the polar head of OA}

As mentionned in section II B, several particle types can be used to describe the polar head of OA, i.e. the carboxylic acid group. No unanimous opinion can be gathered because the polarity of the acidic group depends on the electron donor capacity of the lateral chain ${ }^{14,31}$. In this work, we decided to test several polar head types for the oleic acid, namely types $\mathrm{P}_{1}$ to $\mathrm{P}_{4}$, and to select the one giving the best estimate of the interfacial tension for the water-oleic acid binary. 


\begin{tabular}{c|rrrr} 
System & $n_{\mathrm{GTO}}^{0}$ & $n_{\mathrm{OA}}^{0}$ & $n_{\mathrm{W}}^{0}$ & $x_{\mathrm{OA}}^{0}$ \\
\hline 1 & 1874 & 0 & 15000 & 0 \\
2 & 1780 & 300 & 15000 & 0.144 \\
3 & 1686 & 600 & 15000 & 0.262 \\
4 & 1500 & 1200 & 15000 & 0.444 \\
5 & 1126 & 2400 & 15000 & 0.681 \\
6 & 938 & 3000 & 15000 & 0.762 \\
7 & 750 & 3600 & 15000 & 0.827 \\
8 & 374 & 4800 & 15000 & 0.928 \\
9 & 0 & 6000 & 15000 & 1
\end{tabular}

Table I: Composition of the studied systems. $n_{i}^{0}$ is the initial number of molecules of species $i$ introduced in the simulation. Considering the initial full phase separation, $x_{\mathrm{OA}}^{0}$ is the initial mole fraction of $\mathrm{OA}$ in the oil phase.

We present in figure 4 the interfacial tension at the water-OA interface (system 9) computed from molecular dynamics using different polar head types. The computed values are compared with the experimental data obtained using the DSA apparatus, $11.4 \pm 0.3 \mathrm{mN} \mathrm{m}^{-1}$. It is worth noting that our value is much lower than the one reported by Demond and Lindner ${ }^{32}, 15.59 \mathrm{mN} \mathrm{m}^{-1}$ (see reference 3 therein, from Young and Harkins, 1928). However, it is in good agreement with recent data from Kallio et $a .^{33}, 12.0 \pm 0.5 \mathrm{mN} \mathrm{m}^{-1}$.

As expected, the interfacial tension decreases as the polarity of the polar head increases and get closer to the water bead type polarity. The interfacial tension calculated with particle $\mathrm{P}_{2}$ fits well with the experimental one. Type $\mathrm{P}_{2}$ was thus selected for the rest of the simulations to describe the OA polar head in order to reproduce correctly the water-OA interfacial tension. This result indicates that the polar head in OA is less polar than acetic acid $\left(\mathrm{P}_{3}\right)$ which is consistent with a more important donor character of a long aliphatic carbon chain. 


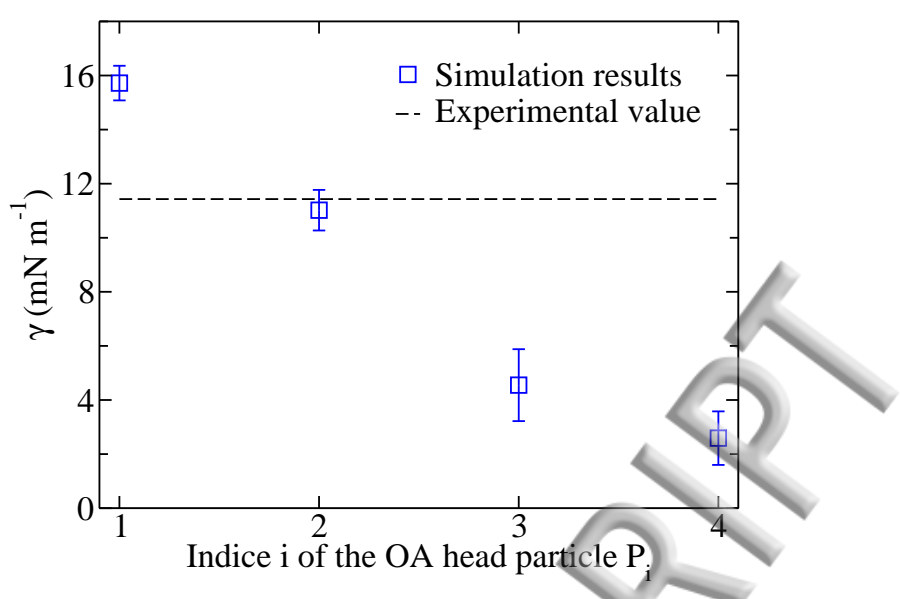

Figure 4: Interfacial tension of the water-OA mixture (system 9) versus the index $i$ of the carboxylic acid polar bead $\mathrm{P}_{i}$. The higher the index $i$, the higher the polarity of the bead.

\section{B. Reaching the equilibrium state}

Before computing any property, (it is mandatory to check that simulation boxes have reached thermodynamic equilibrium. The initial configurations already contain two phases but some time is required before local concentrations reach their final values: depending on the lipid phase composition, OA and GTO molecules leave or enrich the interfaces whereas a few water molecules leave the aqueous phase and solubilize into the lipid phase. The timescale associated with these processes is rather long, even with a coarse-grained force field like MARTINI. To monitor the system behavior during the transient state, the time evolution of the number of water, OA and GTO molecules in the bulk of the lipid region was followed (see below for a precise definition of the bulk lipid region). We present in figure 5 the number of molecules into the bulk region $n_{i}^{b}$. As expected, the number of water molecules in the lipid phase first increases with time, OA molecules leave the bulk lipid region and migrate towards the interfaces while GTO molecules move in the opposite direction. Obviously, this process depends on temperature, phase composition and dimensions of the simulation box; though, as can be seen in figure 5 , the timescale for this process is typically in the range 400-600 ns. The same range is observed for all systems studied. Moreover, the MARTINI force field has been shown to speedup dynamical processes by a factor of four ${ }^{8,13}$, meaning that the "physical" time needed to reach chemical equilibrium in this system can therefore be as large as $2 \mu \mathrm{s}$. 


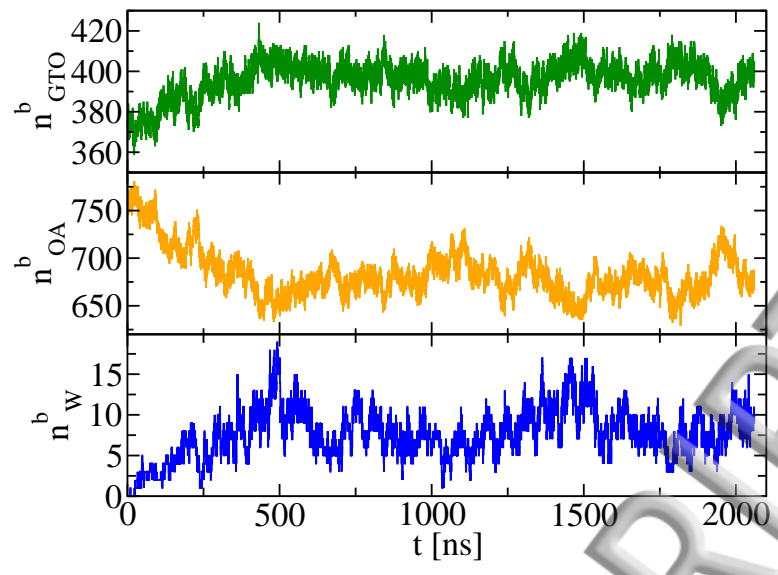

Figure 5: Time evolution of the number of water (W), OA and GTO molecules into the lipid bulk region $n_{i}^{b}$ for system 5 (simulation timescale).

\section{Local organization in the ternary mixtures}

We present in figure 6 the local mass density of bead types in systems 2 and 7, i.e. with low and high OA/GTO ratio, respectively. The mass density profil is built using the position of each bead type along the $z$ axis, normal to the water-oil interfaces. Each full box length is divided into 300 layers of $0.156 \pm 0.001 \mathrm{~nm}$. The local density is thus the mass per layer for each bead divided by the layer volume, averaged over $1.6 \mu$ s data at equilibrium. Water $\left(\mathrm{P}_{4}\right.$ type) and anti-freeze water $\left(\mathrm{BP}_{4}\right.$ type) densities are represented explicitly along with $\mathrm{OA}$ head ( $\mathrm{P}_{2}$ type), OA tail ( $\mathrm{C}_{1}$ and $\mathrm{C}_{3}$ types) beads, GTO ester groups ( $\mathrm{N}_{a}$ type) and GTO tails $\left(\mathrm{C}_{1}\right.$ and $\mathrm{C}_{3}$ types). The total density per layer is also shown.

Three distinct regions can be distinguished: the aqueous region, between layers 0-40 and 260-300, the lipid bulk region between layers 120-180 and two interfacial regions. Aqueous and lipid bulk regions are homogeneous and not perturbed by the presence of the interfaces: the total density profile is flat in the corresponding regions. We also notice that the average density of water in the aqueous region is $0.896 \mathrm{~g} \mathrm{~cm}^{-3}$, very close to the bulk density of water plus anti-freeze water (0.1 bead ratio BP4/P4) obtained by us using independent simulations under the same thermodynamic conditions: $0.898 \mathrm{~g} \mathrm{~cm}^{-3}$. For the lipid bulk region, we did independent simulations of pure OA and pure GTO and obtained equilibrium densities of $0.818 \mathrm{~g} \mathrm{~cm}^{-3}$ and $0.841 \mathrm{~g} \mathrm{~cm}^{-3}$, respectively. These values are identical within $0.1 \%$ to the OA density in system 9 (water-OA) and the GTO density in system 1 (water- 


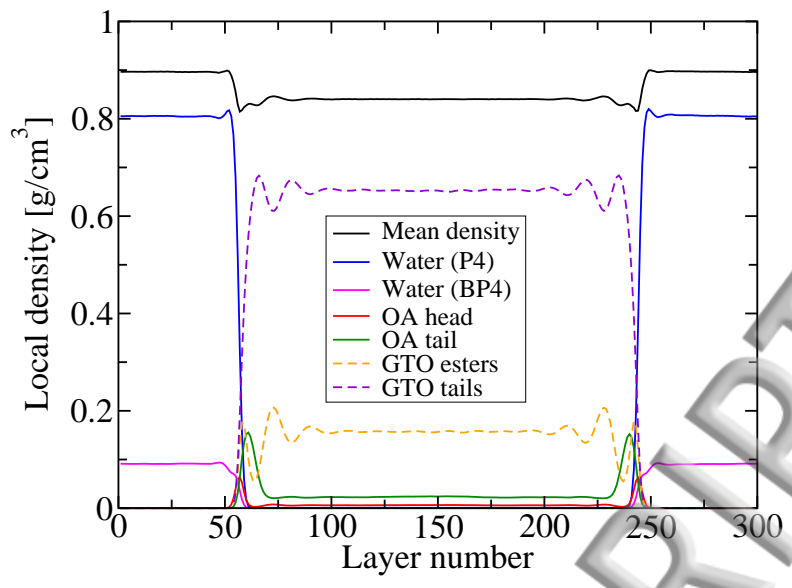

(System 2)

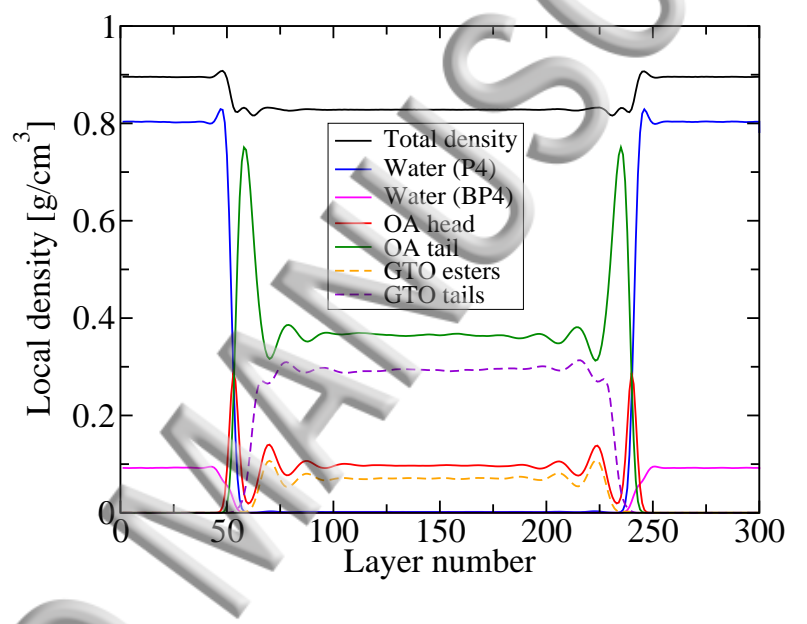

(System 7)

Figure 6: Local mass density profiles of bead types and total density in systems 2 and 7 . Layer width is $0.156 \mathrm{~nm}$.

GTO). Therefore, as the bulk properties are reproduced in the aqueous and lipid regions, the simulation boxes are considered large enough to study at the same time bulk and interfacial properties.

Although computed densities in the water and lipid regions agree with bulk densities of the models, they are notably different from experimental values (by roughly $10 \%$ for water and $8 \%$ for the lipids). This clearly indicates some limitations of the MARTINI force field. A spurious effect was also observed concerning the local organization of $\mathrm{P}_{4}$ versus $\mathrm{BP}_{4}$ beads at the water-oil interface, discussed in the SI. 
PublishingNo lipid molecule is totally dissolved in the aqueous phase whereas several water beads are totally dissolved in the lipid region. A fraction of oleic acid and trioleate molecules are organized at the water-oil interface. The corresponding polar beads, $\mathrm{P}_{2}$ for $\mathrm{OA}$ and $\mathrm{N}_{a}$ for GTO, are in contact with water beads, whereas tail beads $\mathrm{C}_{1}$ and $\mathrm{C}_{3}$ are in contact with the lipid phase only. When OA and GTO are present at the interface, the $\mathrm{P}_{2}$ beads are more in contact with water beads than $\mathrm{N}_{a}$ beads. All ternary mixtures (systems 2 to 8) present the same characteristics. We also notice some interesting structure for the interfacial region. The maxima of local density of polar beads $\mathrm{P}_{2}$ and $\mathrm{N}_{a}$ coincide with the minima of tail beads. A detailed analysis and inspection of the density profile shows that a lipid bilayer is formed by OA and GTO molecules at the water-oil interface whatever the composition of the system, even for pure GTO. This is further confirmed by the distance

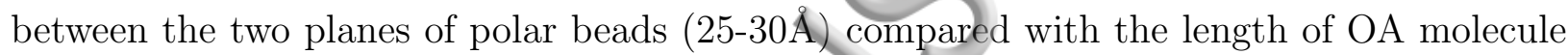

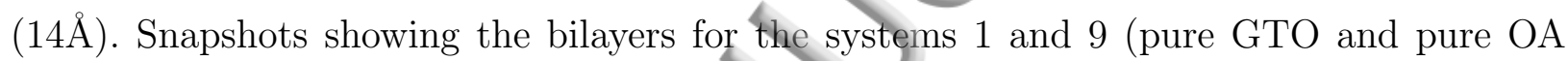
oil phases) are presented in figure 7 and a schematic representation of the bilayers is shown in figure 8. A more quantitative analysis is given in SI, as well as movies of system 5 that demonstrate the bilayer presence, the large occupation of the interface by OA molecules, and the exchange of OA and GTO between the bulk and the interfacial layer.

\section{Lipid phase composition}

As mentioned earlier, water, OA and GTO local molar fractions change with time until some equilibrium is reached. The equilibrium state is characterized, in particular, by the molar fraction of the different species in the lipid bulk phase $x_{\mathrm{i}}^{b}$ (layers 120-180), i.e. the region far from the water-oil interfaces (equation 5).

Because of the limited number of molecules in the simulation box, the OA (and GTO) molar fraction in the lipid bulk region, $x_{\mathrm{OA}}^{\mathrm{b}}$ can be significantly different from the initial molar fraction $x_{\mathrm{OA}}^{0}$ (equation 2) and from the interfacial molar fraction $x_{\mathrm{OA}}^{\text {int }}$ (equation 6).

$$
\begin{aligned}
x_{\mathrm{OA}}^{\mathrm{b}} & =\frac{n_{\mathrm{OA}}^{\mathrm{b}}}{n_{\mathrm{OA}}^{\mathrm{b}}+n_{\mathrm{GTO}}^{\mathrm{b}}} \\
x_{\mathrm{OA}}^{\mathrm{int}} & =\frac{n_{\mathrm{OA}}^{\mathrm{int}}}{n_{\mathrm{OA}}^{\mathrm{int}}+n_{\mathrm{GTO}}^{\mathrm{int}}}
\end{aligned}
$$



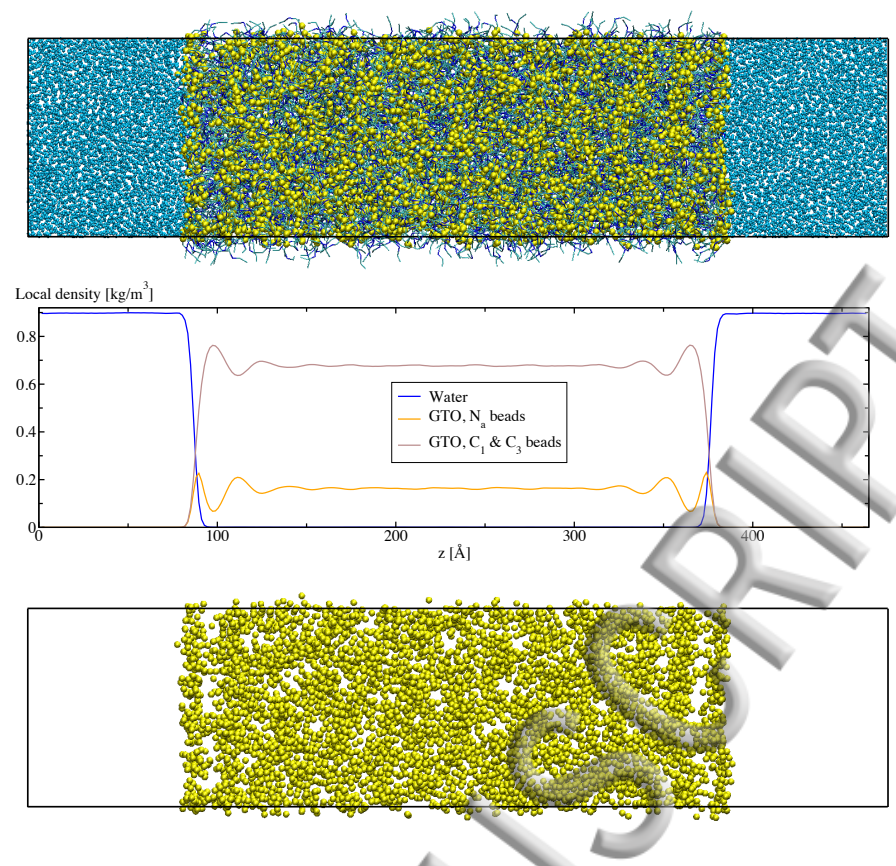

(a) pure GTO

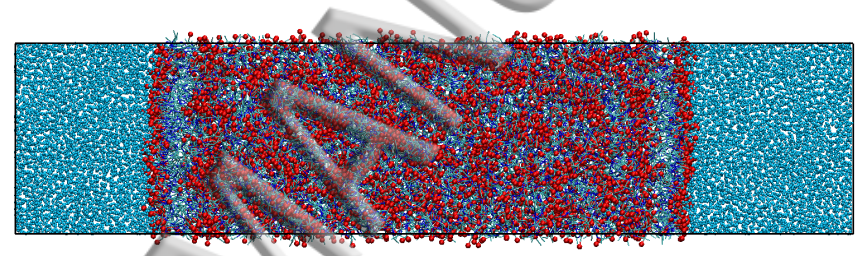

Local density $\left[\mathrm{kg} / \mathrm{m}^{3}\right]$
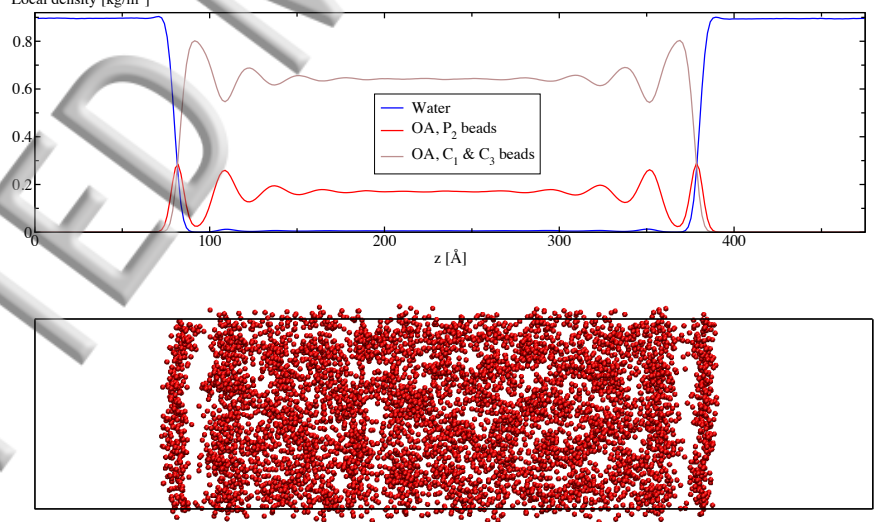

(b) pure OA

Figure 7: Snapshots of the bilayer at the water-oil interface in the systems 1 containing pure GTO oil phase (a) and 9 containing pure OA oil phase (b). The snapshots with all the beads and with only the polar beads $\left(\mathrm{P}_{2}\right.$ or $\left.\mathrm{N}_{\mathrm{a}}\right)$ are compared to the density profiles of the corresponding simulation. Snapshots produced thanks to VMD software ${ }^{34,35}$ 


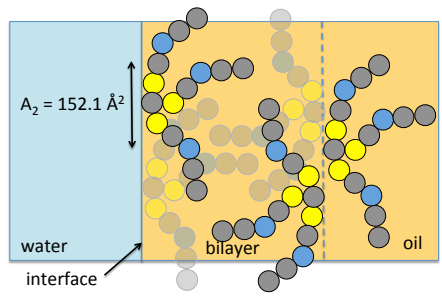

(a) pure GTO

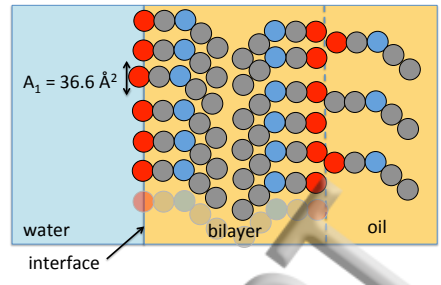

(b) pure OA

Figure 8: Graphical representation of the bilayer at the water-oil interface for the system 1 containing pure GTO (a) and the system 9 containing pure OA (b)

This is not true for macroscopic systems were the number of molecules in the bulk phase is much larger than the number of molecules at the interfaces. In macroscopic systems, the bulk phase serves as a reservoir for OA (and GTO) molecules and $x_{\mathrm{OA}}^{\mathrm{b}} \approx x_{\mathrm{OA}}^{0}$. Therefore, care should be taken to account for this difference, when comparing interfacial tension (or other composition dependent properties) obtained from molecular simulations with experimental data. We report table II the computed OA mole fraction in the lipid bulk phase and compare it with the initial value for each system. It is important to note that, although OA is not a surfactant molecule, the final bulk concentration is always smaller than the initial concentration, especially at low OA composition.

We present in figure 9 the estimation of water solubility in the lipid bulk phase as a function of OA molar fraction. Computed values are compared with measurements from Platford $^{36}$ and Chiou ${ }^{37}$. Experimental data give water solubility in pure OA and pure GTO in the range $283-310 \mathrm{~K}$. They show that water is much more soluble in OA than GTO and also that solubility strongly depends on temperature: according to Platford ${ }^{36}$, solubility in OA changes from $0.13 \mathrm{~mol} \mathrm{~L}^{-1}$ at $283 \mathrm{~K}$ to $0.26 \mathrm{~mol} \mathrm{~L}^{-1}$ at $310 \mathrm{~K}$ and solubility in GTO changes from 0.051 to $0.1 \mathrm{~mol} \mathrm{~L} \mathrm{~L}^{-1}$ over the same temperature range. Water solubility in GTO given by Chiou is $0.056 \mathrm{~mol} \mathrm{~L}^{-1}$ at $298 \mathrm{~K}$, smaller than Platford value of 0.072 at $293 \mathrm{~K}$. A single data point in a OA-GTO mixture, at $x_{O A}^{b} \approx 0.75$ is provided by Platford, at $310 \mathrm{~K}$.

As can be seen in figure 9, molecular simulation data are in fair agreement with experi- 


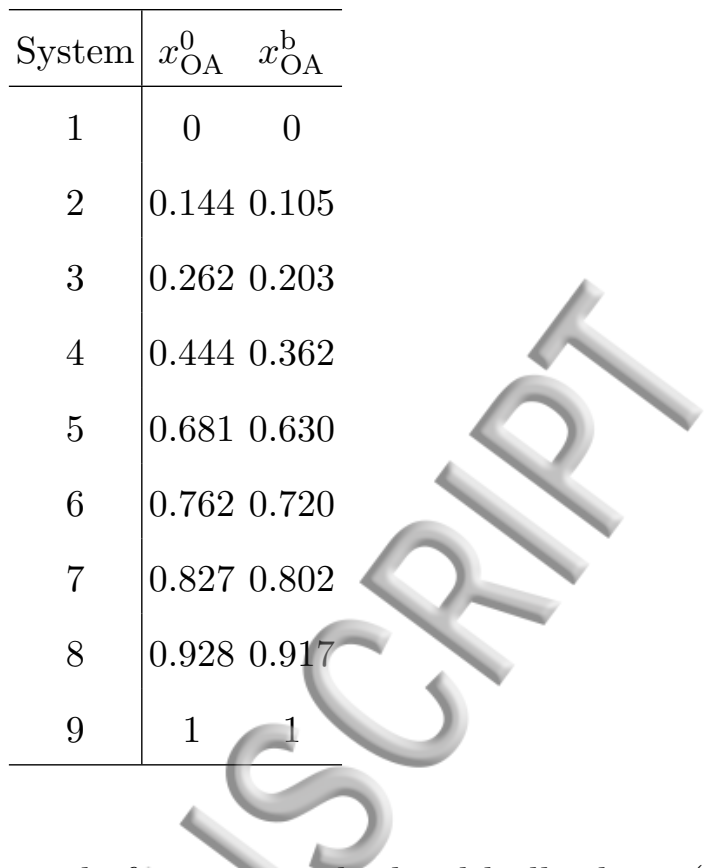

Table II: Initial and equilibrium OA mole fraction in the lipid bulk phase (layers 120-180).

Figure 9: Water concentration versus OA concentration in the lipid bulk phase. Molecular simulation data are compared with experimental measurements from Platford ${ }^{36}$ and

$$
\mathrm{Chiou}^{37}
$$

ments. Water solubility is lower in GTO than OA. Molecular simulation data overestimate water solubility in OA and underestimate water solubility in GTO. The solubility value in the OA-GTO mixture is not correctly predicted by simulation. Platford measured a solubility close to the one in pure OA at $310 \mathrm{~K}$. Molecular simulations clearly show that water solubility strongly increases with OA concentration, though only for $x_{\mathrm{OA}}^{b}$ larger than 0.65. 


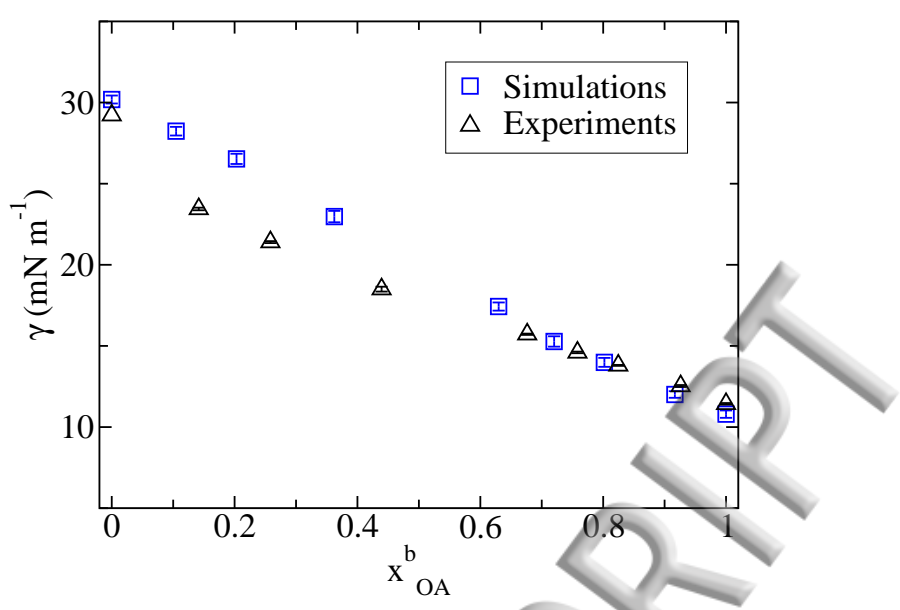

Figure 10: Measured and computed interfacial tension $\gamma$, vérsus OA molar fraction in the bulk region $x_{\mathrm{OA}}^{\mathrm{b}}$

Clearly, more experimental data at intermediate concentration would be required to confirm the water solubility values in $\mathrm{OA}-\mathrm{GTO}$ mixtures.

\section{E. Interfacial tension and area per molecule analysis}

We present in figure 10 the computed interfacial tension between water and OA-GTO mixtures versus OA molar fraction in the bulk region. The interfacial tension $\gamma$ decreases linearly with increasing OA concentration in the organic phase. This is consistent with the amphiphilic properties of $\mathrm{OA}$ due to the polar head and the apolar tail. A good agreement is observed between computed and experimental data shown in figure 10 for OA molar fraction

above 0.6. For $0<x_{\mathrm{OA}}^{\mathrm{b}}<0.6$, the interfacial tension is overestimated by up to $15 \%$. However $\gamma$ for water-GTO and water-OA systems are well predicted: the computed interfacial tensions for GTO and OA are respectively $30.2 \pm 0.3 \mathrm{mN} \mathrm{m}^{-1}$ and $10.8 \pm 0.3 \mathrm{mN} \mathrm{m}^{-1}$, with corresponding experimental values of $29.2 \pm 0.1 \mathrm{mN} \mathrm{m}^{-1}$ and $11.4 \pm 0.1 \mathrm{mN} \mathrm{m}^{-1}$. The disagreement observed at low OA concentration suggests that OA-GTO interactions are not well described, leading to incorrect concentrations at interfaces: OA concentration at interfaces should be larger in order to recover experimental data.

We tried to compare these values with theoretical predictions. There exists only a few methods to predict interfacial tension data for multicomponent mixtures. From a practical point of view, the Fu et al. method ${ }^{38}$ is one of the most employed for ternary mixtures. It is 
Publishiligs on a thermodynamic relationship established by Shain and Prausnitz ${ }^{39}$. Using several assumptions, Fu et al. were able to relate interfacial tension to the mutual solubilities of the different species. Since OA or GTO and water are almost immiscible, the mutual solubilities can hardly be measured or even more computed. It is therefore difficult to estimate $\gamma$ in the ternary systems from data obtained using simulation. Instead, we computed interfacial tension of binary systems, assuming that OA and GTO concentration in water is zero. The interfacial tension can thus be obtained from:

$$
\gamma=-\ln \left(x_{w}^{o}\right) \frac{K R T}{A_{w}}
$$

where $x_{w}^{o}$ is the water molar fraction in the oil phase, $K=0.9414$ is a constant proposed by Fu et al., $R$ is the perfect gas constant, $T$ the temperature and $A_{w}=35.6 \AA^{2}$ per molecule, the Van der Waals surface area of the water molecule. Using water concentrations issued from molecular simulations, we obtain $\gamma_{\mathrm{w}-\mathrm{OA}}=25.1 \mathrm{mN} \mathrm{m}^{-1}$ and $\gamma_{\mathrm{w}-\mathrm{GTO}}=37.8 \mathrm{mN} \mathrm{m}^{-1}$. It is clear that the model proposed by Fu et al. is in disagreement with a direct computation of interfacial tensions. This may come from an incorrect value for $K$. As evidenced by Santos et $a l .{ }^{40}$, the constant $K$ can differ from the one proposed by $\mathrm{Fu}$ et al., depending on the type of mixtures investigated. Clearly, precise molecular simulations on model systems could help to check the $\mathrm{Fu}$ et al. equation.

Although the computed interfacial tension is not in full agreement with measurements, we believe that these simulations contain realistic informations about the local structure at the oil-water interface. The number of OA and GTO molecules in the interfacial region were computed using the local density profiles of $\mathrm{P}_{2}$ and $\mathrm{N}_{a}$ beads, by direct integration of their first peak at the oil-water interface. From our simulations on systems 1 and 9, the area occupied by OA and GTO at the interface between pure product and water are respectively $A_{\mathrm{OA}}^{*}=36.6 \AA^{2}$ and $A_{\mathrm{GTO}}^{*}=152.1 \AA^{2}$. The results are compared to Langmuir balance experiments that were described in the literature for OA and $\mathrm{GTO}^{41-43}$ and complementary experimental results (figure 11). The principle of these experiments is to spread lipid molecules as a monolayer on an water-air interface and to compress this interface with barriers. According to literature, a film of pure OA presents a compressed liquid organization with an area per molecule between 20 and $50 \AA^{2}$. A film of pure GTO presents an expended liquid phase with an area per molecule between 100 and $150 \AA^{2}$. The film collapses for lower area per molecule. In the simulation, in presence of a majority of OA at the interface, the 


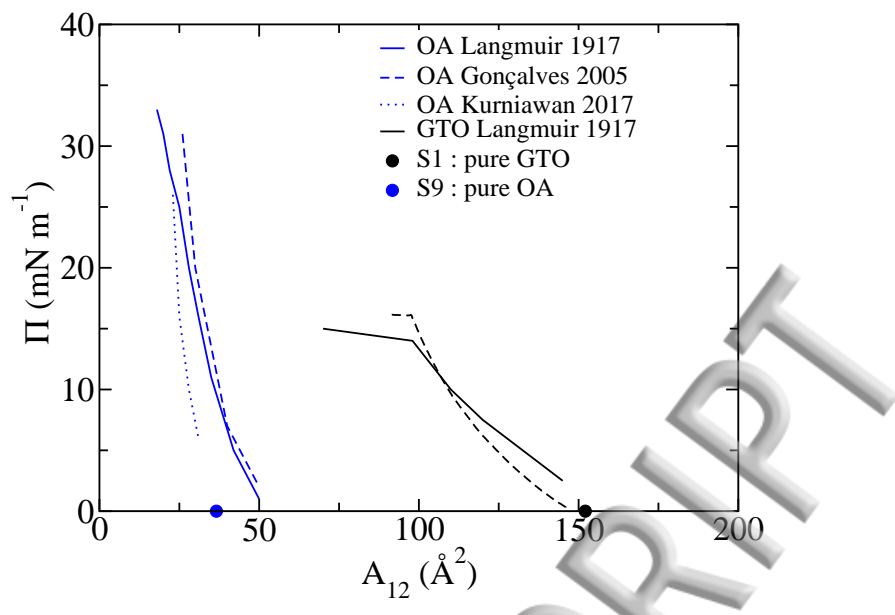

Figure 11: Surface pressure $\Pi$ at the water-oil interface versus the area per molecule $A_{i}$. compared to experimental data obtained with pure-OA and pure GTO ${ }^{41-43}$

area is $36.6 \AA^{2}$. This result corresponds to a condensed liquid phase and correlate to the experimental results. Considering the simulations with pure GTO, the area is $152.1 \AA^{2}$, similar to the experimental data in the same conditions and an expended liquid phase is present.

We present in figure 12 the evolution of the global area per molecule, $A$, along with the excess area per molecule, $A_{\text {ex }}$ defined as:

$$
A_{\mathrm{ex}}=A-\left[x_{\mathrm{OA}}^{\mathrm{int}} A_{\mathrm{OA}}^{*}+\left(1-x_{\mathrm{OA}}^{\mathrm{int}}\right) A_{\mathrm{GTO}}^{*}\right]
$$

where the term in braquets is an ideal contribution, $A^{*}$, and $x_{\mathrm{OA}}^{\mathrm{int}}$ represents the OA molar fraction at the interface. The excess area presents a small positive deviation, showing that the interface is slightly less compact than ideal product. The excess area reaches $5 \AA^{2}$ for $x_{\mathrm{OA}}^{\mathrm{int}}=0.891 /$ However, the small values observed show that a rather good mixing of OA and GTO occurs at the interface, which seems realistic with identical hydrophobic tails.

\section{CONCLUSION}

Nine systems containing oleic acid and triolein mixtures in contact with water were simulated using a coarse grained molecular simulation and the MARTINI force field. The polar bead $\mathrm{P}_{2}$ was chosen to represent the fatty acid head in order to reproduce the experimental interfacial tension. 


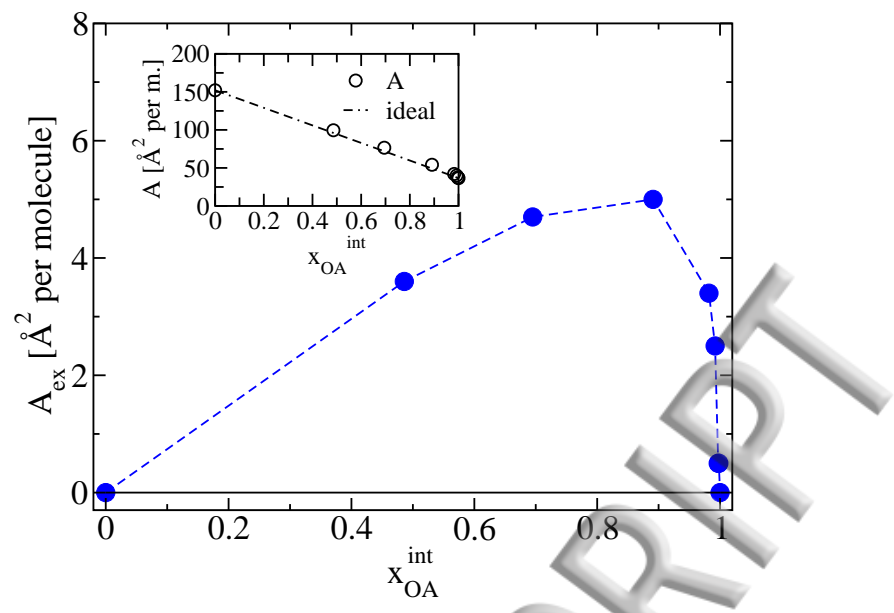

Figure 12: Excess surface per molecule, $A_{\mathrm{ex}}$, versus $x_{\mathrm{OA}}^{\mathrm{int}}$, the molar fraction of $\mathrm{OA}$ at the interface. Insert: area per molecule $A$ and ideal mixture $A^{*}$ contributions.

Large simulation boxes $\left(107 \times 107 \times 465 \AA^{3}\right)$ are required to ensure that the two present interfaces have no interaction. The simulations ran during $2000 \mathrm{~ns}, 500 \mathrm{~ns}$ to reach chemical equilibrium and 1500 ns to produce trajectories along which interfacial tension, water solubility and local densities were computed. If we account for the dynamical speedup (roughly 4) introduced by the MARTINI force field, the physical time needed to reach equilibrium is $2 \mu s$ and the production runs last for $6 \mu \mathrm{s}$. Such long timescales support the use of CG simulations. Inspection of density profiles suggests that a bilayer structure of the lipids exists at the water-oil interface.

Computed interfacial tensions are close to experimental values with a small deviation of maximum $15 \%$ at low OA concentrations. The water solubility in the lipid phase is in fair agreement with experiments, between 0.03 and $0.32 \mathrm{~g} \mathrm{~L}^{-1}$, rising with the OA content. The surface area occupied by OA and GTO fit with experimental data; under simulation conditions OA organization at the interface is that of a compressed liquid whereas GTO behaves like an expended liquid.

Through all these results the usefulness of CG simulations for the analysis of water-oil interfacial organization is demonstrated. This method will bring local informations on the organization of target compounds, for example in biomass fractionation processes or food additives formulations.

Also, we are quite confident that this CG model can be extended to the prediction of interfacial tension and water solubility of fatty acids and triglycerides with different chain 
Publishileggths (e.g. palmitic, arachidic, linoleic acids, and the triglyceride derived from these fatty acids). These quantities can be useful in order to derive new theoretical models which are really scarce in this area (water, fatty acids, triglycerides).

\section{SUPPLEMENTARY MATERIAL}

See supplementary material to obtain details on the force filed parameters, the behaviour of $\mathrm{P}_{4}$ and $\mathrm{BP}_{4}$ at the oil-water interface, a deepened analysis of the lipid organisation demonstrating the bilayer organization, movies that illustrate the bilayer and the evolution of local OA and GTO composition at the interface versus OA mole fraction in the oil phase.

\section{ACKNOWLEDGMENTS}

This work was supported by the CNRS program ENRS projets fédérateurs 2014. The simulations were performed using high-performance computing resources from CCIPL (Centre de Calcul Intensif des Pays de la Loire), France.

\section{REFERENCES}

${ }^{1}$ E. Clavijo, Liliana Villafaña López, Shuli Liu, P. Bourseau, M. Frappart, C. Monteux, and Estelle Couallier. Understanding microalgae lipids recovery by membrane processes: cross flow filtration of a representative synthetic mixture (o/w emulsion). 10th World Congress of Chemical Engineering (WCC10), 2017.

${ }^{2}$ Christelle Lopez, Valérie Briard-Bion, Olivia Ménard, Eric Beaucher, Florence Rousseau, Jacques Fauquant, Nadine Leconte, and Benoit Robert. Fat globules selected from whole milk according to their size: Different compositions and structure of the biomembrane, revealing sphingomyelin-rich domains. Food Chemistry, 125(2):355-368, 2011.

${ }^{3}$ Claire C. Berton-Carabin, Marie Hélène Ropers, and Claude Genot. Lipid oxidation in oil-in-water emulsions: Involvement of the interfacial layer. Comprehensive Reviews in Food Science and Food Safety, 13:945-977, 2014.

${ }^{4}$ R. J. Gillams, T. Nylander, T. S. Plivelic, M. K. Dymond, and G. S. Attard. Formation of inverse topology lyotropic phases in dioleoylphosphatidylcholine/oleic acid and 
Publishingioleoylphosphatidylethanolamine/oleic acid binary mixtures. Langmuir, 30(12):3337-44, 2014.

${ }^{5}$ Thad A. Harroun, Norbert Kučerka, Mu-Ping Nieh, and John Katsaras. Neutron and xray scattering for biophysics and biotechnology: examples of self-assembled lipid systems. Soft Matter, 5(14):2694, 2009.

${ }^{6}$ Åge a Skjevik, Benjamin D. Madej, Callum J. Dickson, Knut Teigen, Ross C. Walker, and Ian R. Gould. All-atom lipid bilayer self-assembly with the amber and charmm lipid force fields. Chem. Commun., 51(21):4402-4405, 2015.

${ }^{7}$ Multiscale modelling of soft matter. Faraday Discuss., 144:3-7, 2010.

${ }^{8}$ Siewert J. Marrink and D. Peter Tieleman. Perspective on the martini model. Chemical Society Reviews, 42(16):6801, 2013.

${ }^{9}$ Riccardo Baron, Daniel Trzesniak, Alex H. de Vries, Andreas Elsener, Siewert J. Marrink, and Wilfred F. van Gunsteren. Comparison of thermodynamic properties of coarse-grained and atomic-level simulation models. ChemPhys Chem, 8(3):452-461, feb 2007.

${ }^{10}$ Tristan Bereau and Kurt Kremer. Automated parametrization of the coarse-grained martini force field for small organic molecules. Journal of Chemical Theory and Computation, 11(6):2783-2791, 2015.

${ }^{11}$ Helgi I. Ingolfsson, Cesar A. Lopez, Jaakko J. Uusitalo, Djurre H. de Jong, Srinivasa M. Gopal, Xavier Periole, and Siewert J. Marrink. The power of coarse graining in biomolecular simulations. Wiley Interdisciplinary Reviews-Computational Molecular Science, 4(3):225 248, 2014.

${ }^{12}$ Siewert J. Marrink, Alex H. de Vries, and Alan E. Mark. Coarse grained model for semiquantitative lipid simulations. The Journal of Physical Chemistry B, 108(2):750-760, jan 2004.

${ }^{13}$ Siewert J. Marrink, H. Jelger Risselada, Serge Yefimov, D. Peter Tieleman, and Alex H. de Vries. The MARTINI force field: coarse grained model for biomolecular simulations. The Journal of Physical Chemistry B, 111(27):7812-7824, jul 2007.

${ }^{14}$ Luca Monticelli, Senthil K. Kandasamy, Xavier Periole, Ronald G. Larson, D. Peter Tieleman, and Siewert-Jan Marrink. The MARTINI coarse-grained force field: Extension to proteins. Journal of Chemical Theory and Computation, 4(5):819-834, may 2008.

${ }^{15}$ Cesar A. Lopez, Zofie Sovova, Floris J. van Eerden, Alex H. de Vries, and Siewert J. Marrink. Martini force field parameters for glycolipids. Journal of Chemical Theory and 
${ }^{16}$ Jean-Claude Neyt, Aurélie Wender, Véronique Lachet, Aziz Ghoufi, and Patrice Malfreyt. Quantitative predictions of the interfacial tensions of liquid-liquid interfaces through atomistic and coarse grained models. Journal of Chemical Theory and Computation, 10(5):18871899, 2014.

${ }^{17}$ Makha Ndao, Julien Devémy, Aziz Ghoufi, and Patrice Malfreyt. Coarse-Graining the Liquid-Liquid Interfaces with the MARTINI Force Field: How Is the Interfacial Tension Reproduced? Journal of Chemical Theory and Computation, 11(8):3818-3828, 2015.

${ }^{18}$ Y. Rotenberg, L. Boruvka, and W. Neumann. Determination of surface tension and contact angle from shapes of axisymmetric fluid interfaces. Journal of Colloid and Interface Science, 93(1):169-183, 1983.

${ }^{19}$ B. Song and J. Springer. Determination of interfacial tension from the profile of a pendant drop using computer-aided image processing. Journal of Colloid and Interface Science, 184:64-76, 1996.

${ }^{20}$ W.F. Drew Bennett, Alexander W. Chen, Serena Donnini, Gerrit Groenhof, and D. Peter Tieleman. Constant pH simulations with the coarse-grained MARTINI model - application to oleic acid aggregates, Canadian Journal of Chemistry, 91(9):839-846, sep 2013.

${ }^{21}$ J. J. Janke, W. F. Bennett, and D. P. Tieleman. Oleic acid phase behavior from molecular dynamics simulations. Langmuir, 30(35):10661-7, 2014.

${ }^{22}$ S. J. Marrinck. Martini - Coarse Grain Forcefield for Biomolecules, http://cgmartini.nl/. ${ }^{23}$ Timo Vuorela, Andrea Catte, Perttu S. Niemelä, Anette Hall, Marja T. Hyvönen, SiewertJan Marrink, Mikko Karttunen, and Ilpo Vattulainen. Role of lipids in spheroidal high density lipoproteins. PLoS Computational Biology, 6(10):e1000964, oct 2010.

${ }^{24}$ Steve Plimpton. LAMMPS web homepage: http://lammps.sandia.gov.

${ }^{25}$ L. Martínez, R. Andrade, E. G. Birgin, and J. M. Martínez. PACKMOL: A package for building initial configurations for molecular dynamics simulations. Journal of Computational Chemistry, 30(13):2157-2164, oct 2009.

${ }^{26}$ Wataru Shinoda, Motoyuki Shiga, and Masuhiro Mikami. Rapid estimation of elastic constants by molecular dynamics simulation under constant stress. Physical Review B, 69(13):134103-1-134103-8, apr 2004.

${ }^{27}$ John G. Kirkwood and Frank P. Buff. The statistical mechanical theory of surface tension. The Journal of Chemical Physics, 17(3):338-343, mar 1949. 
Publishitîg gakenobu Nakamura, Shuhei Kawamoto, and Wataru Shinoda. Precise calculation of the local pressure tensor in Cartesian and spherical coordinates in LAMMPS. Computer Physics Communications, 190:120-128, may 2015.

${ }^{29}$ J. H. Irving and John G. Kirkwood. The Statistical Mechanical Theory of Transport Processes. IV. The Equations of Hydrodynamics. The Journal of Chemical Physics, 18(6):817$829,1950$.

${ }^{30}$ J. P. R. B. Walton, D. J. Tildesley, J. S. Rowlinson, and J. R. Henderson. The pressure tensor at the planar surface of a liquid. Molecular Physics, 48(6):1357-1368, apr 1983.

${ }^{31}$ Djurre H. de Jong, Gurpreet Singh, W. F. Drew Bennett, Clement Arnarez, Tsjerk A. Wassenaar, Lars V. Schäfer, Xavier Periole, D. Peter Tieleman, and Siewert J. Marrink. Improved parameters for the martini coarse-grained protein force field. Journal of Chemical Theory and Computation, 9(1):687-697, jan 2013.

${ }^{32}$ Avery H. Demond and Angela S. Lindner. Estimation of interfacial tension between organic liquids and water. Environmental Science \& Technology, 27(12):2318-2331, 1993.

${ }^{33}$ Kallio Timo, Janne Laine, and Per Stenius. Intermolecular interactions and the adhesion of oleic acid. Journal of Dispersion Science and Technology, 30(2):222-230, 2009.

${ }^{34}$ William Humphrey, Andrew Dalke, and Klaus Schulten. VMD - Visual Molecular Dynamics. Journal of Molecular Graphics, 14:33-38, 1996.

${ }^{35}$ John Stone. An Efficient Library for Parallel Ray Tracing and Animation. Master's thesis, Computer Science Department, University of Missouri-Rolla, April 1998.

${ }^{36}$ R.F. Platford. The octanol-water partitioning of some hydrophobic and hydrophilic compounds. Chemosphere, 12(7-8):1107-1111, jan 1983.

${ }^{37}$ Cary T. Chiou. Partition coefficients of organic compounds in lipid-water systems and correlations with fish bioconcentration factors. Environmental Science 85 Technology, 19(1):57-62, jan 1985.

${ }^{38} \mathrm{Jufu}$ Fu, Buqiang Li, and Zihao Wang. Estimation of fluid-fluid interfacial tensions of multicomponent mixtures. Chemical Engineering Science, 41(10):2673 - 2679, 1986.

${ }^{39}$ S. A. Shain and J. M. Prausnitz. Thermodynamics and interfacial tension of multicomponent liquid-liquid interfaces. AIChE Journal, 10(5):766-773, 1964.

${ }^{40}$ B.M.S Santos, A.G.M Ferreira, and I.M.A Fonseca. Surface and interfacial tensions of the systems water + n-butyl acetate + methanol and water + n-pentyl acetate + methanol at 303.15 k. Fluid Phase Equilibria, 208(1-2):1-21, 2003. 
Publishiftg. Langmuir. The constitution and fundamental properties of solids ans liquids. ii. liquids. Journal of the American Chemical Society, 39:1848-1906, 1917.

${ }^{42}$ A. M. Goncalves da Silva and R. I. Romao. Mixed monolayers involving dppc, dodab and oleic acid and their interaction with nicotinic acid at the air-water interface. Chem Phys Lipids, 137(1-2):62-76, 2005.

${ }^{43}$ J. Kurniawan, K. Suga, and T. L. Kuhl. Interaction forces and membrane charge tunability: Oleic acid containing membranes in different ph conditions. Biochim Biophys Acta, 1859(2):211-217, 2017. 


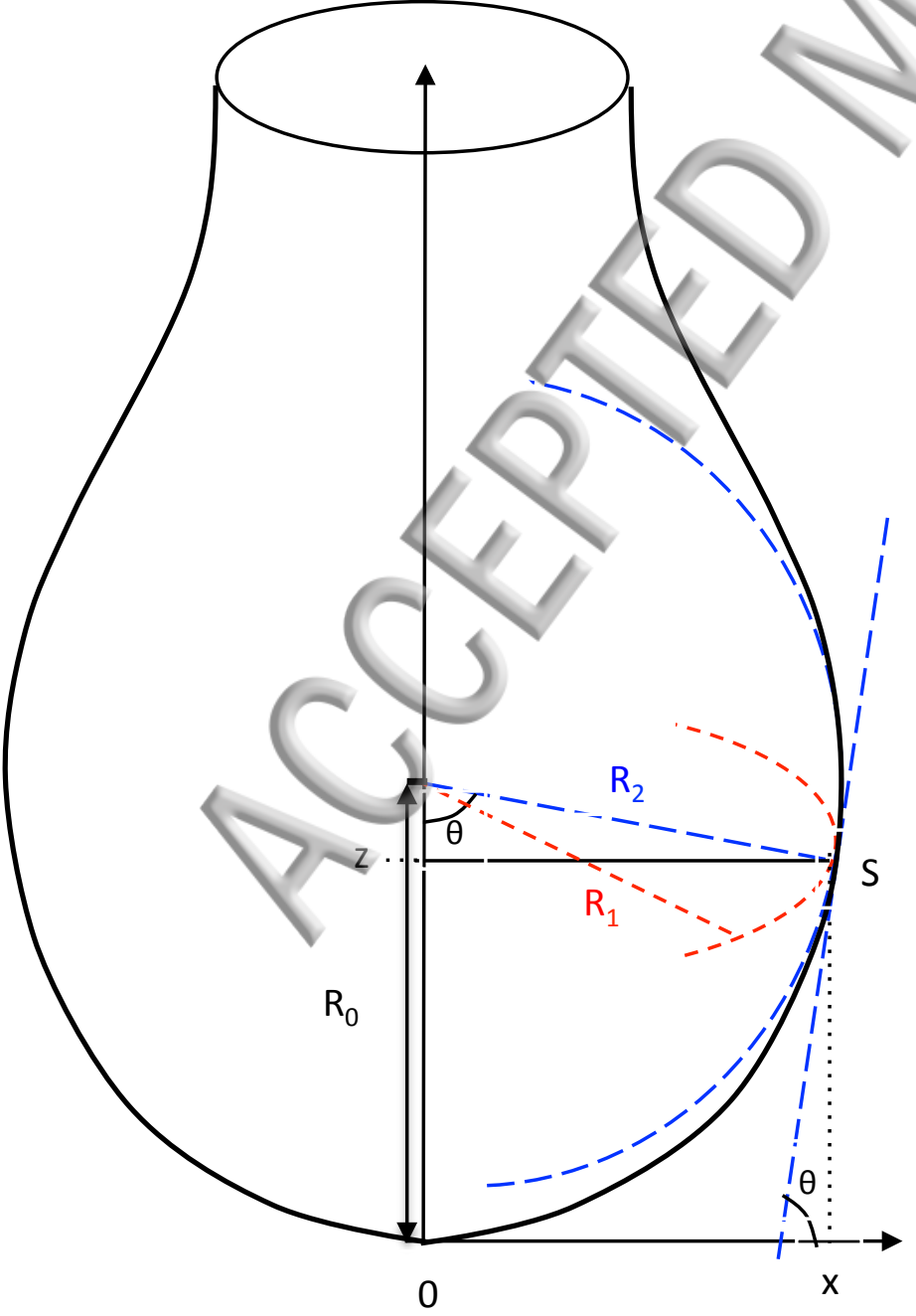




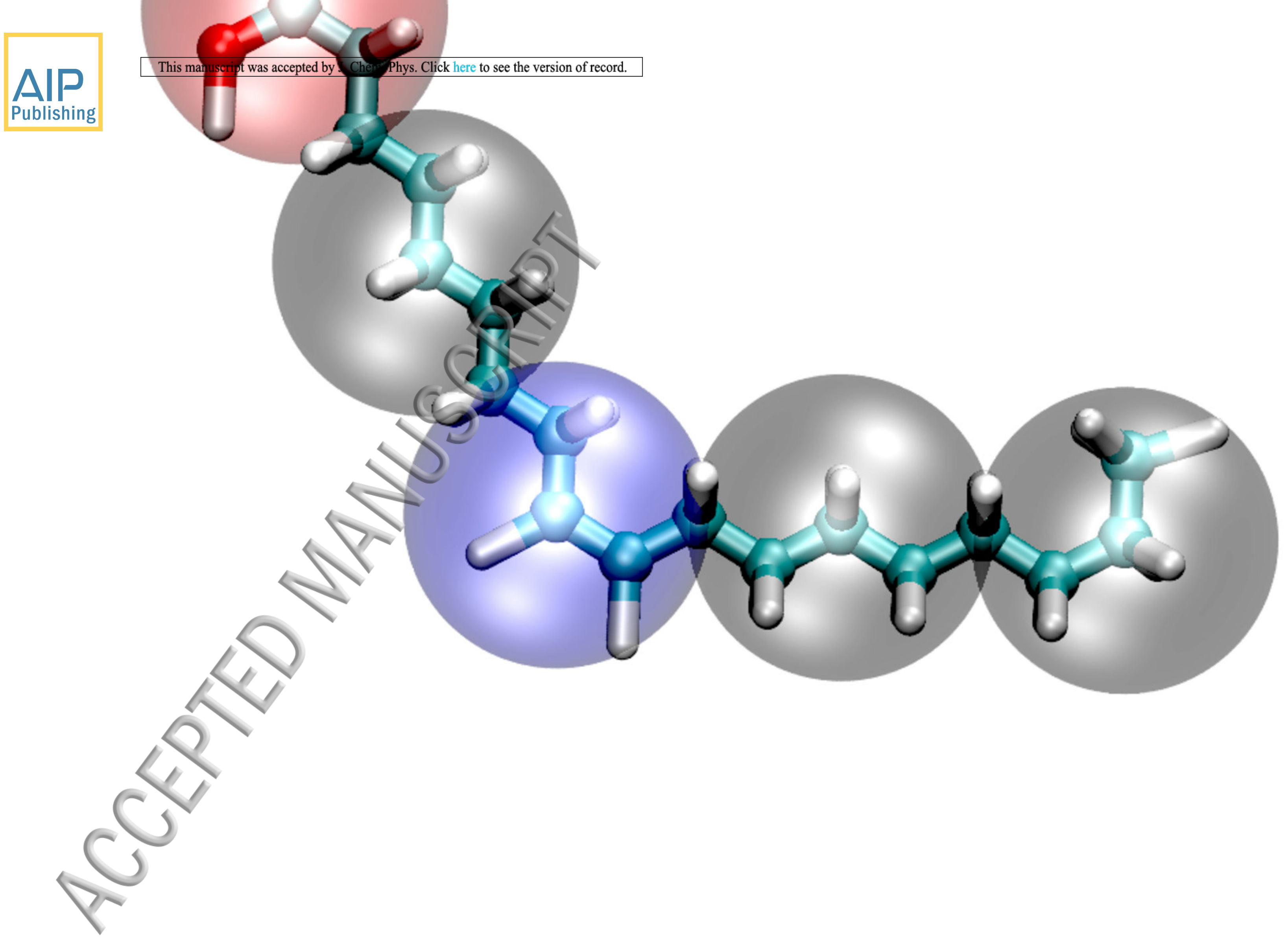




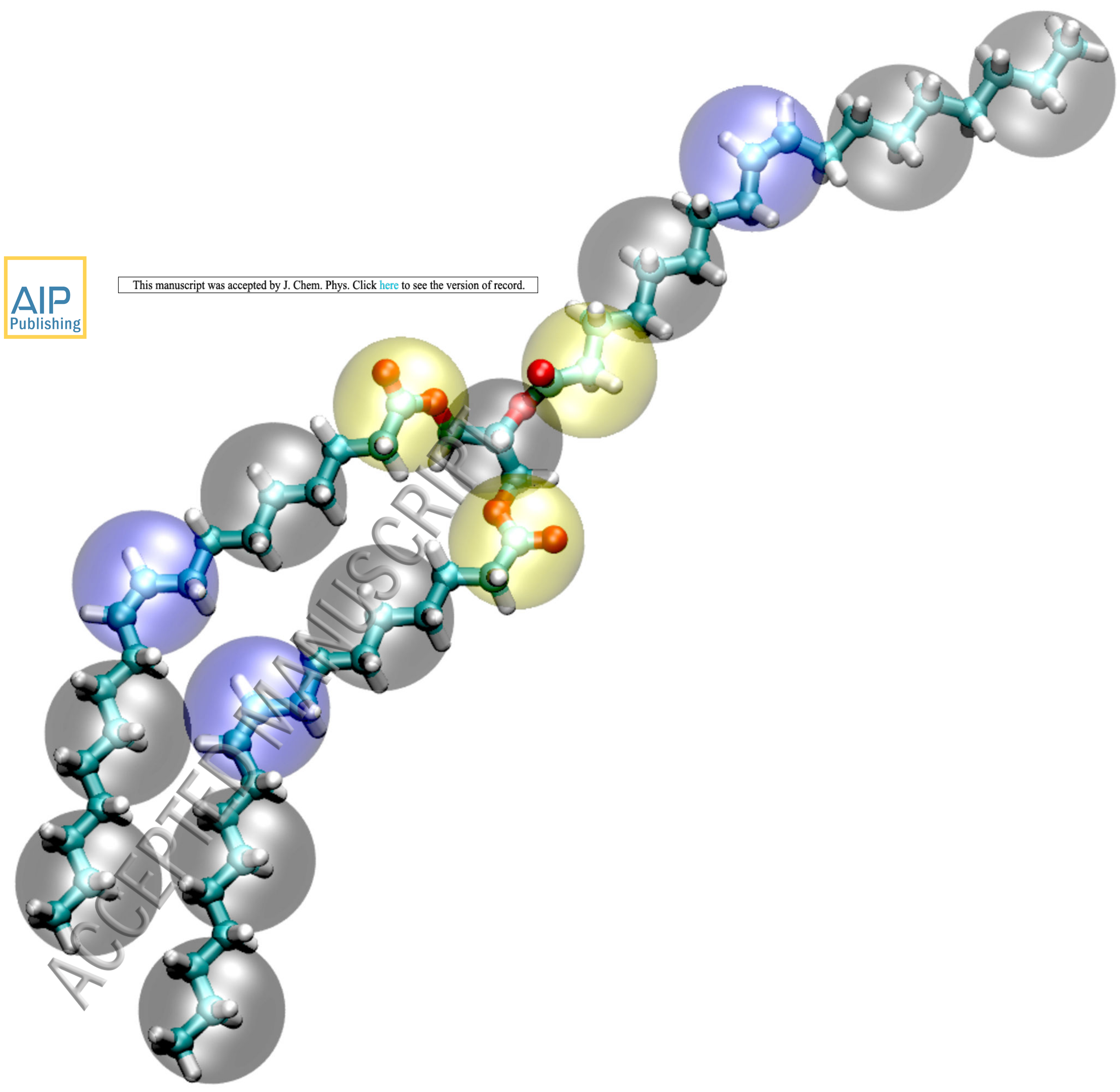




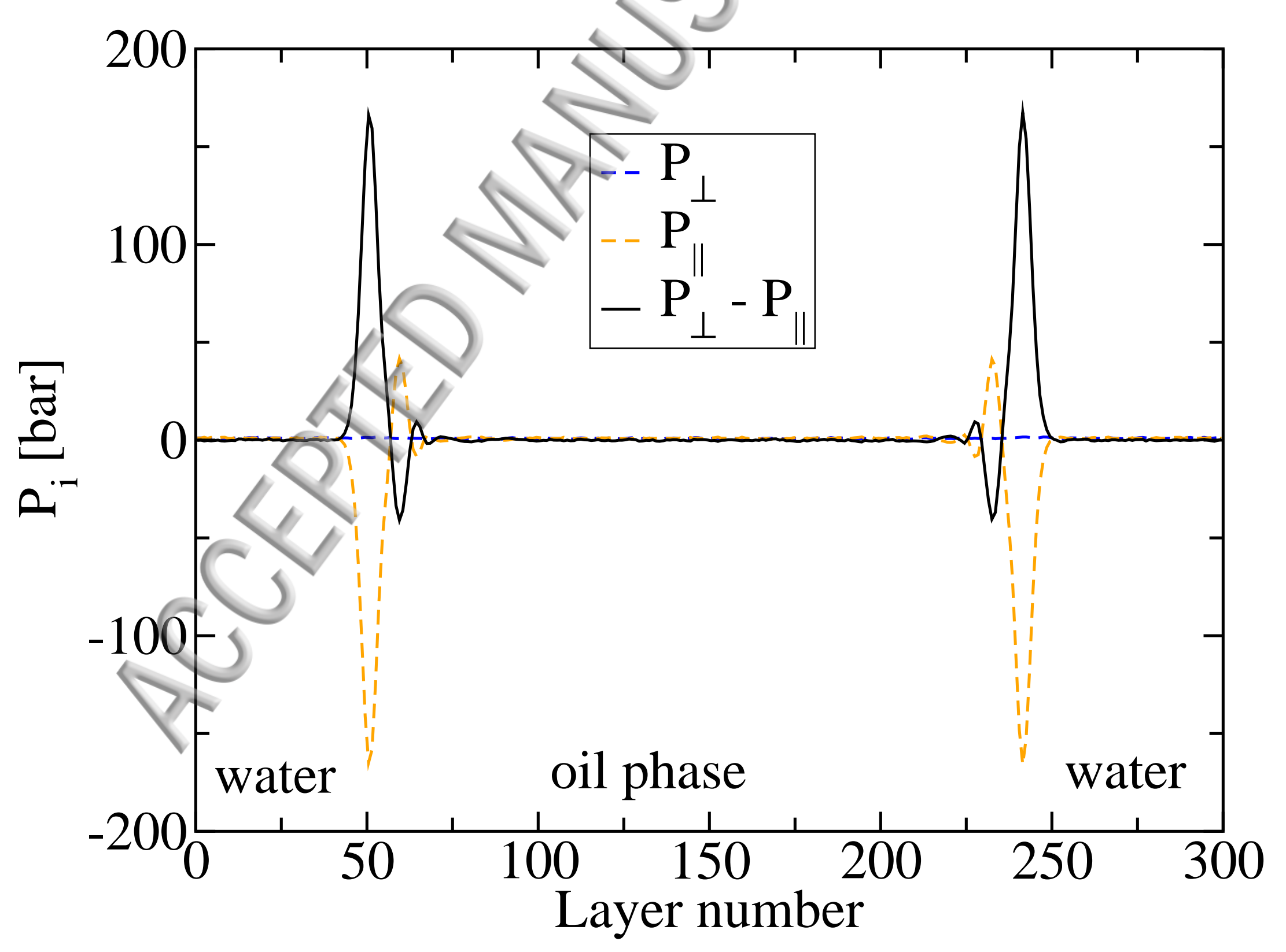




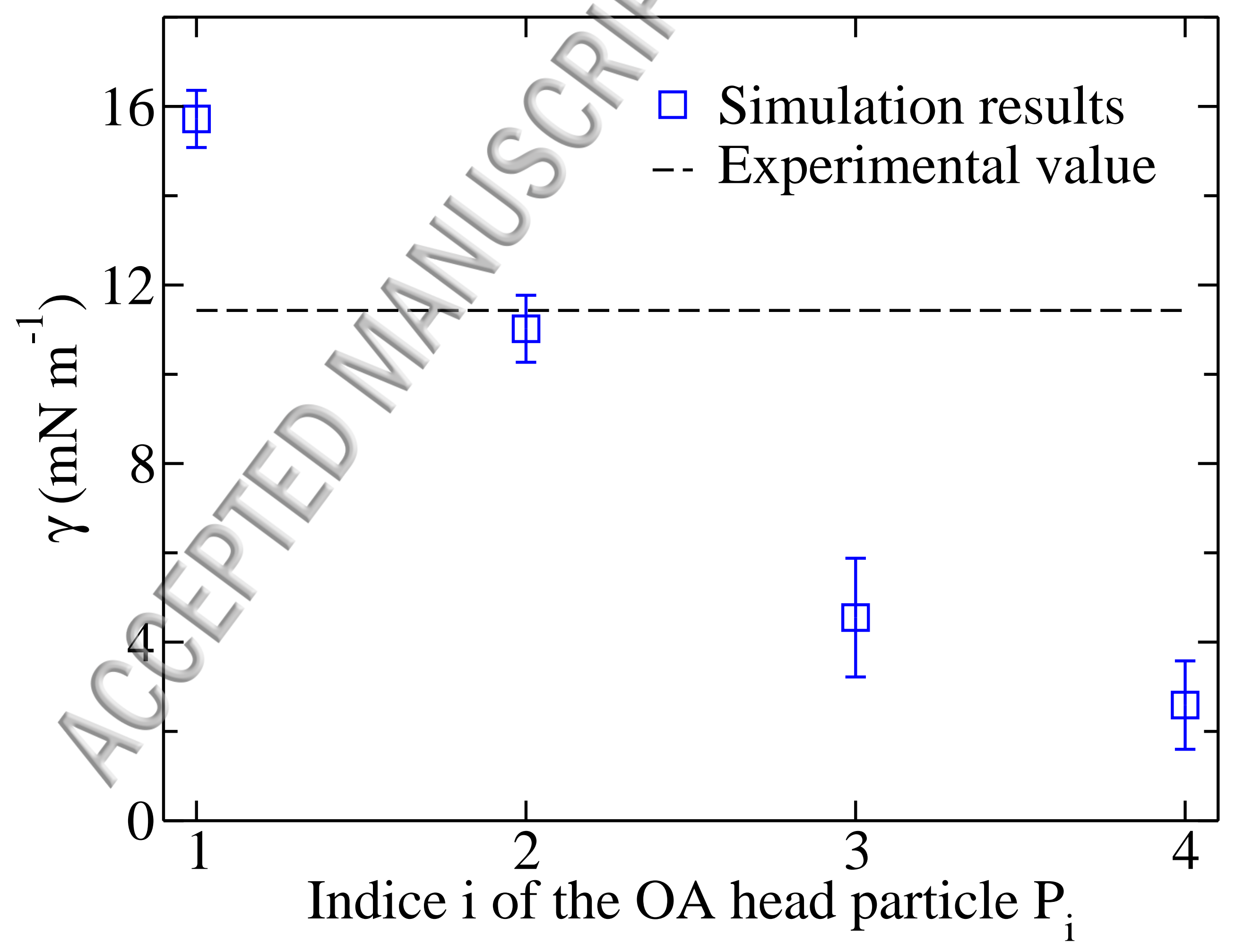




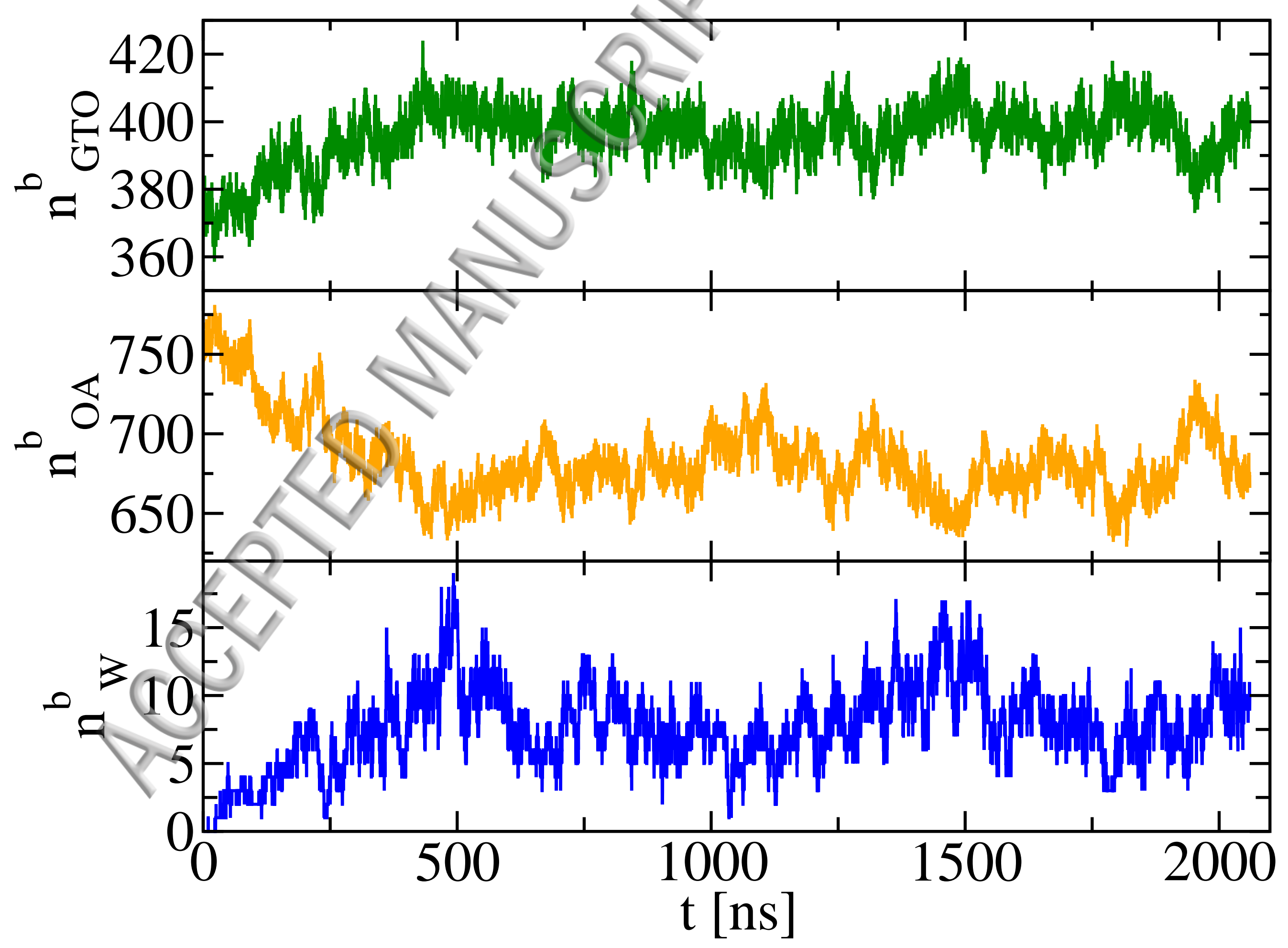




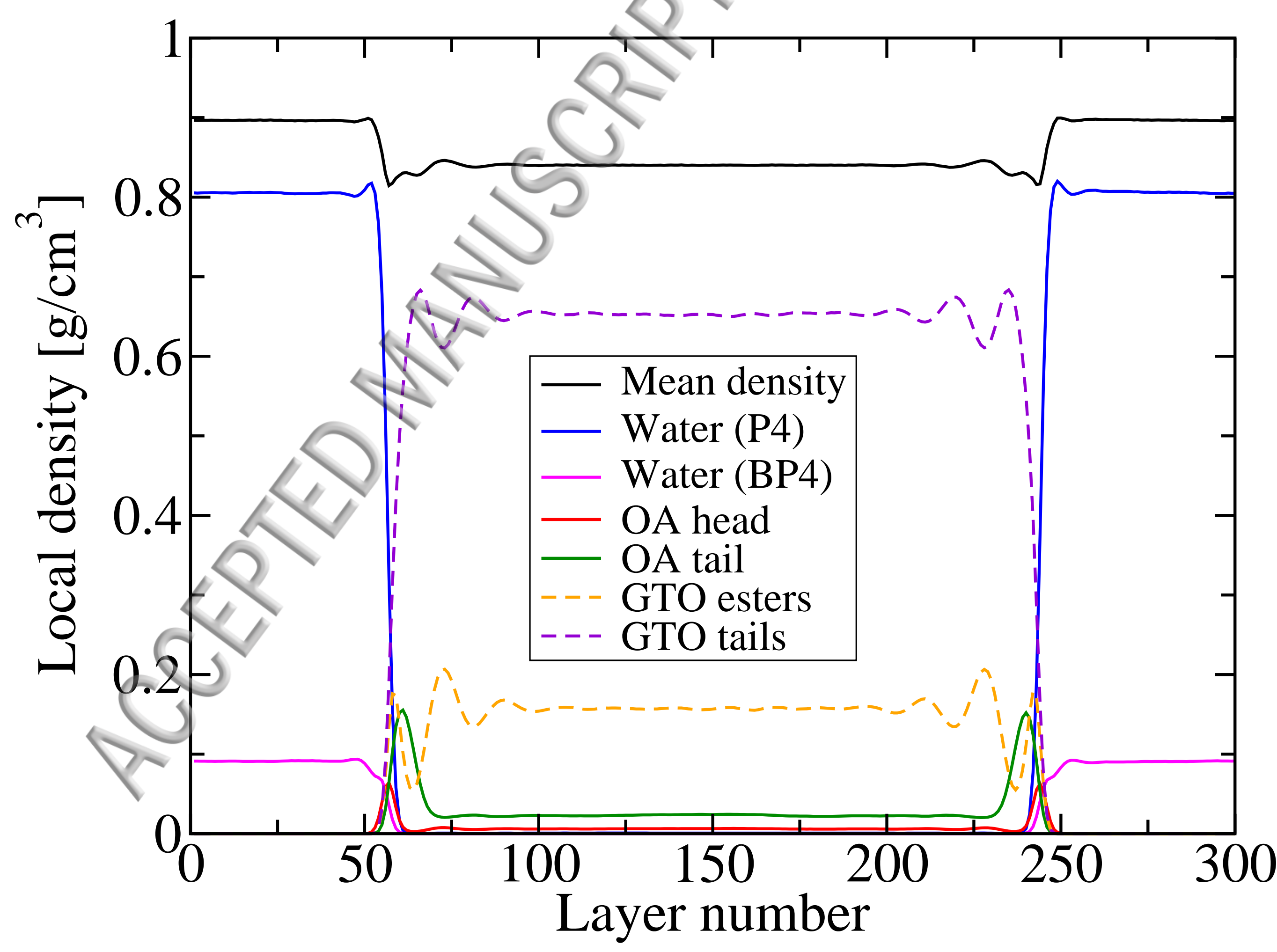




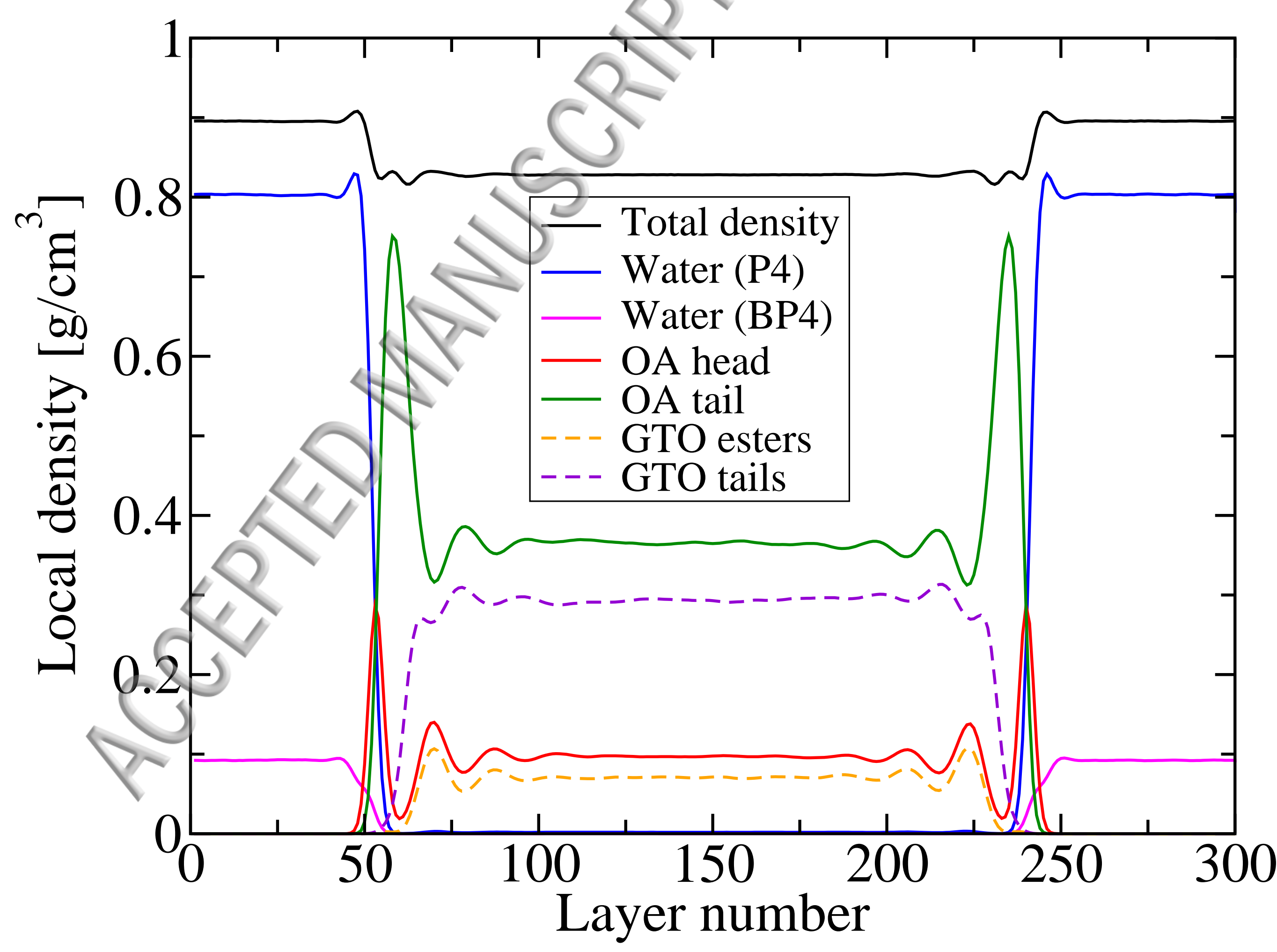




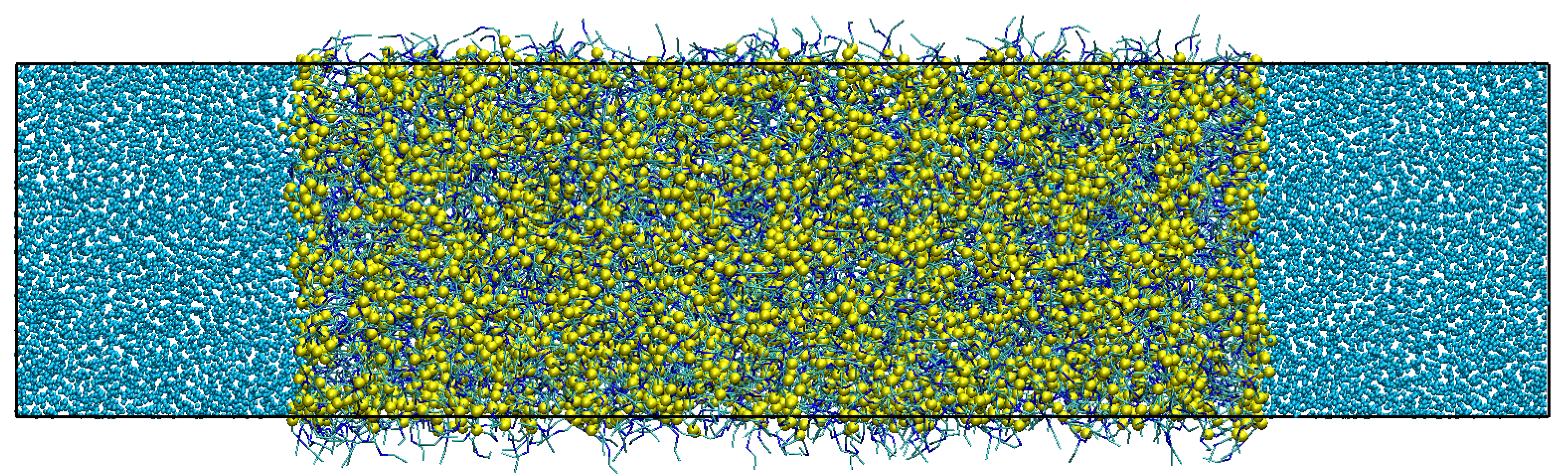

Local density $\left[\mathrm{kg} / \mathrm{m}^{3}\right]$

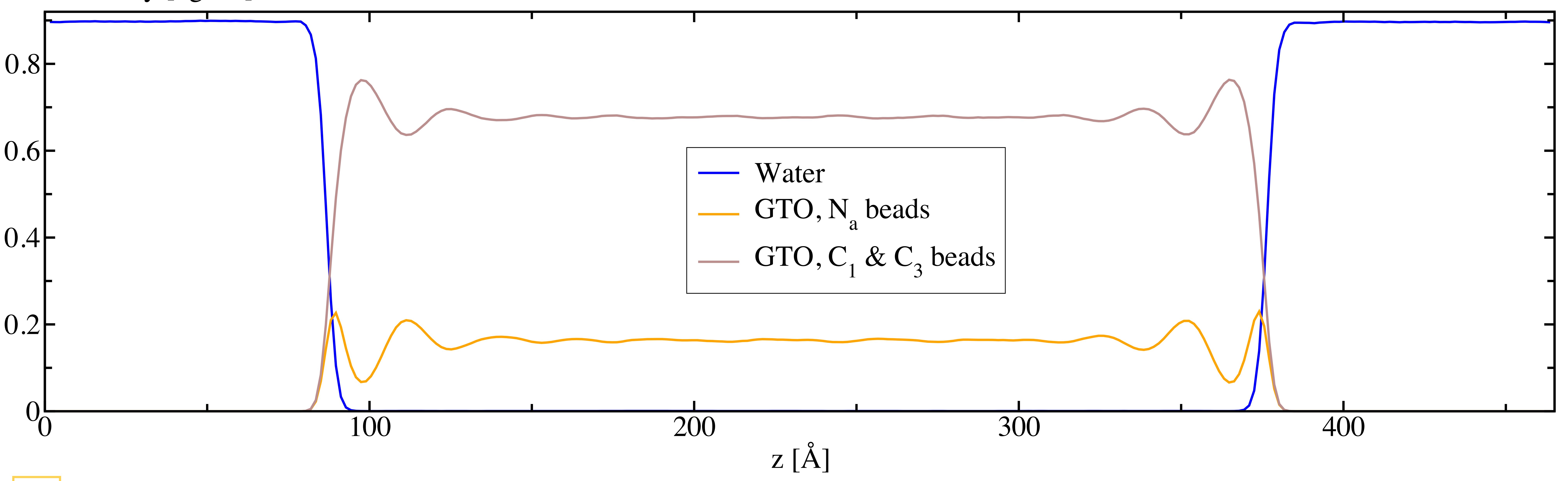

Alp

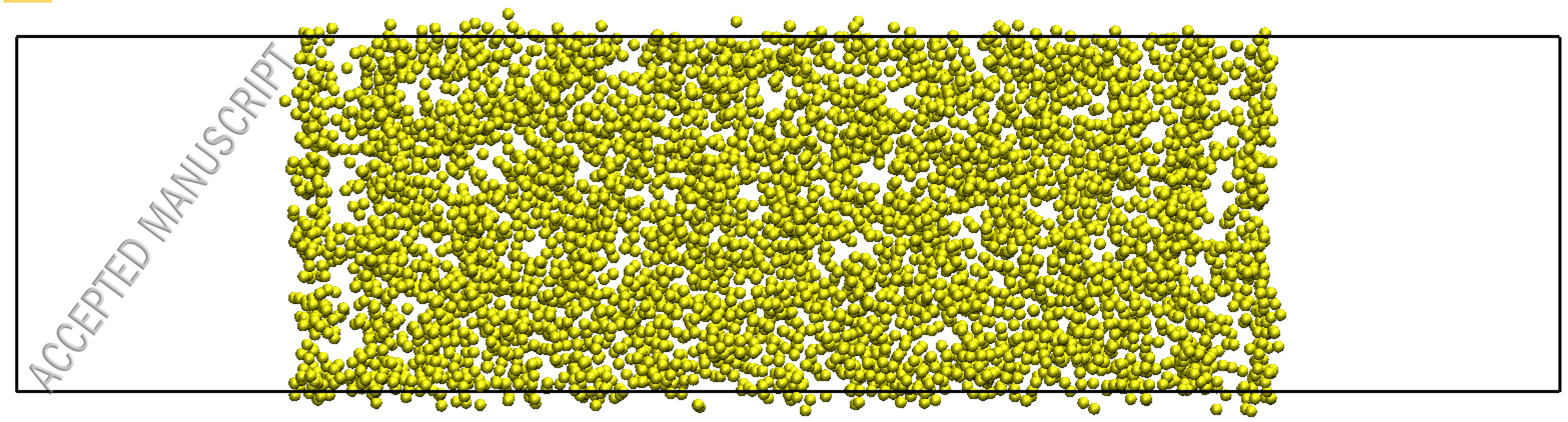




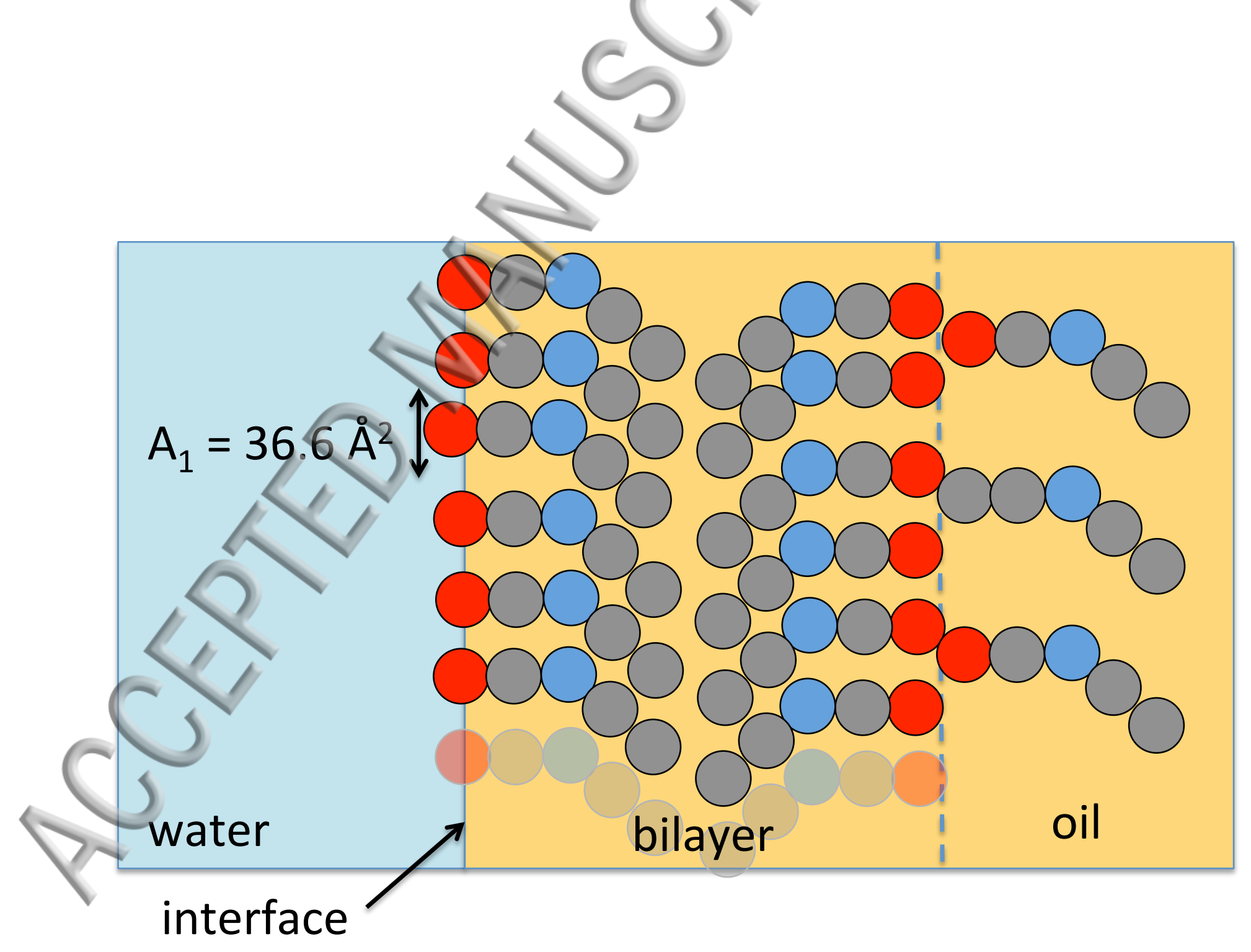




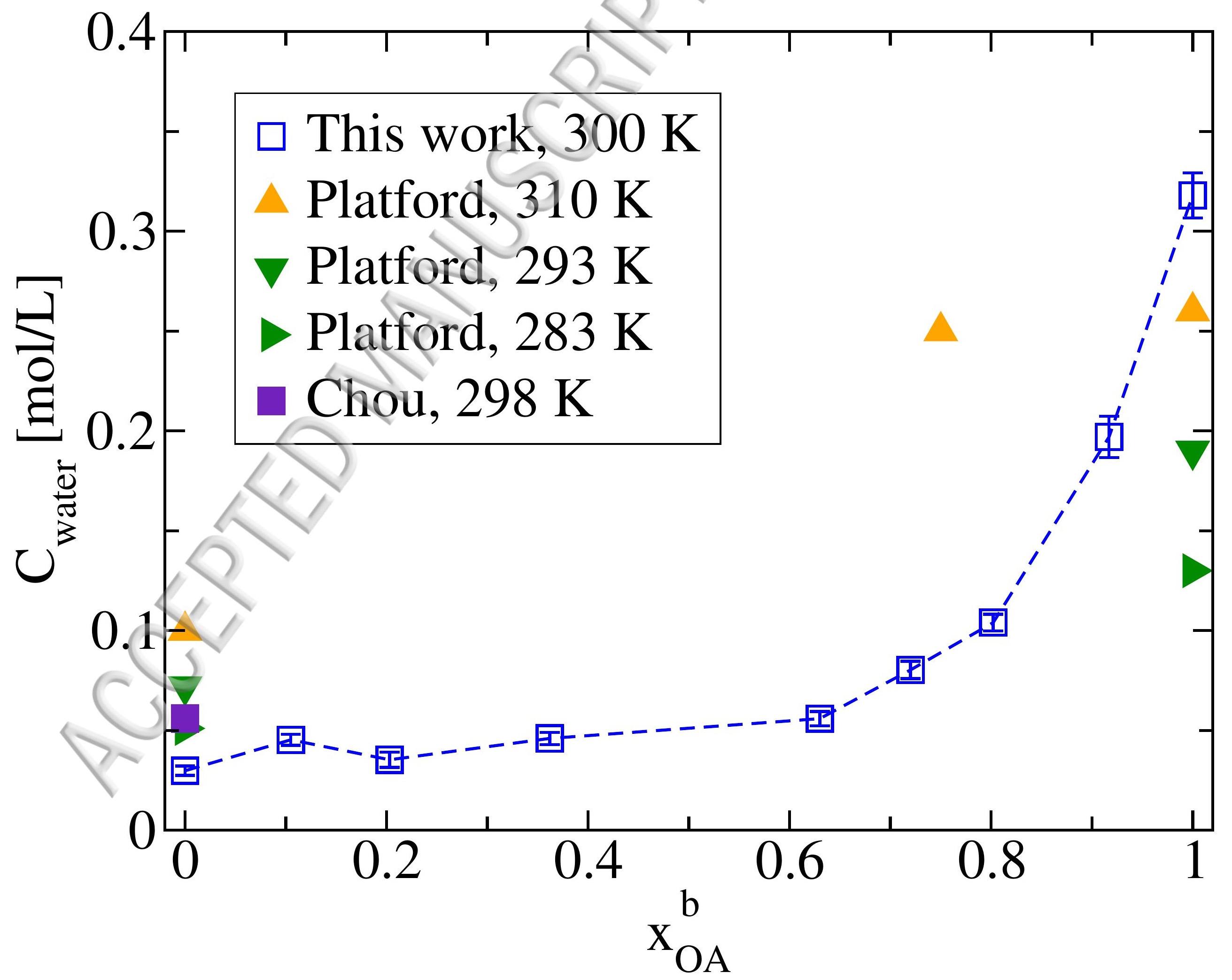




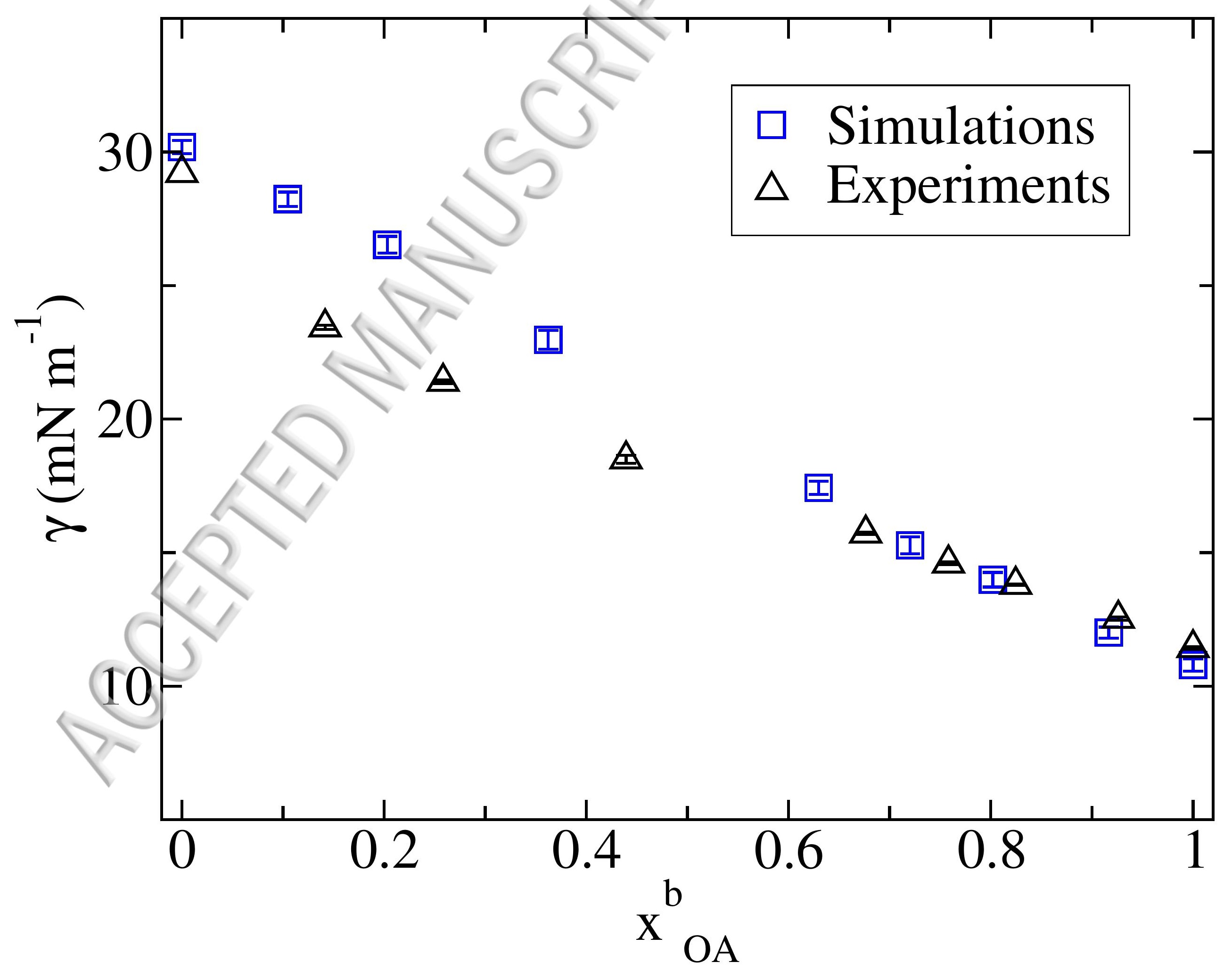




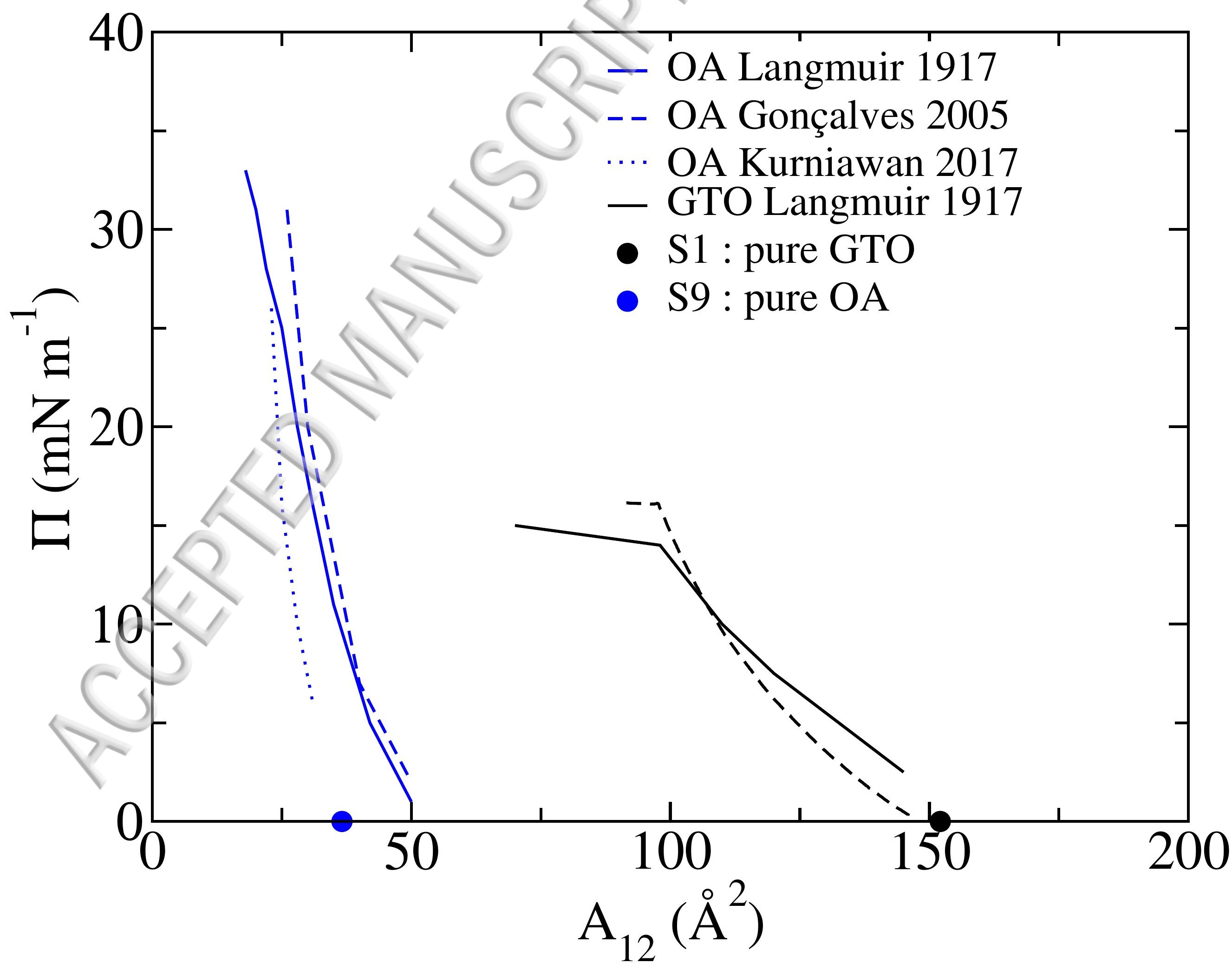




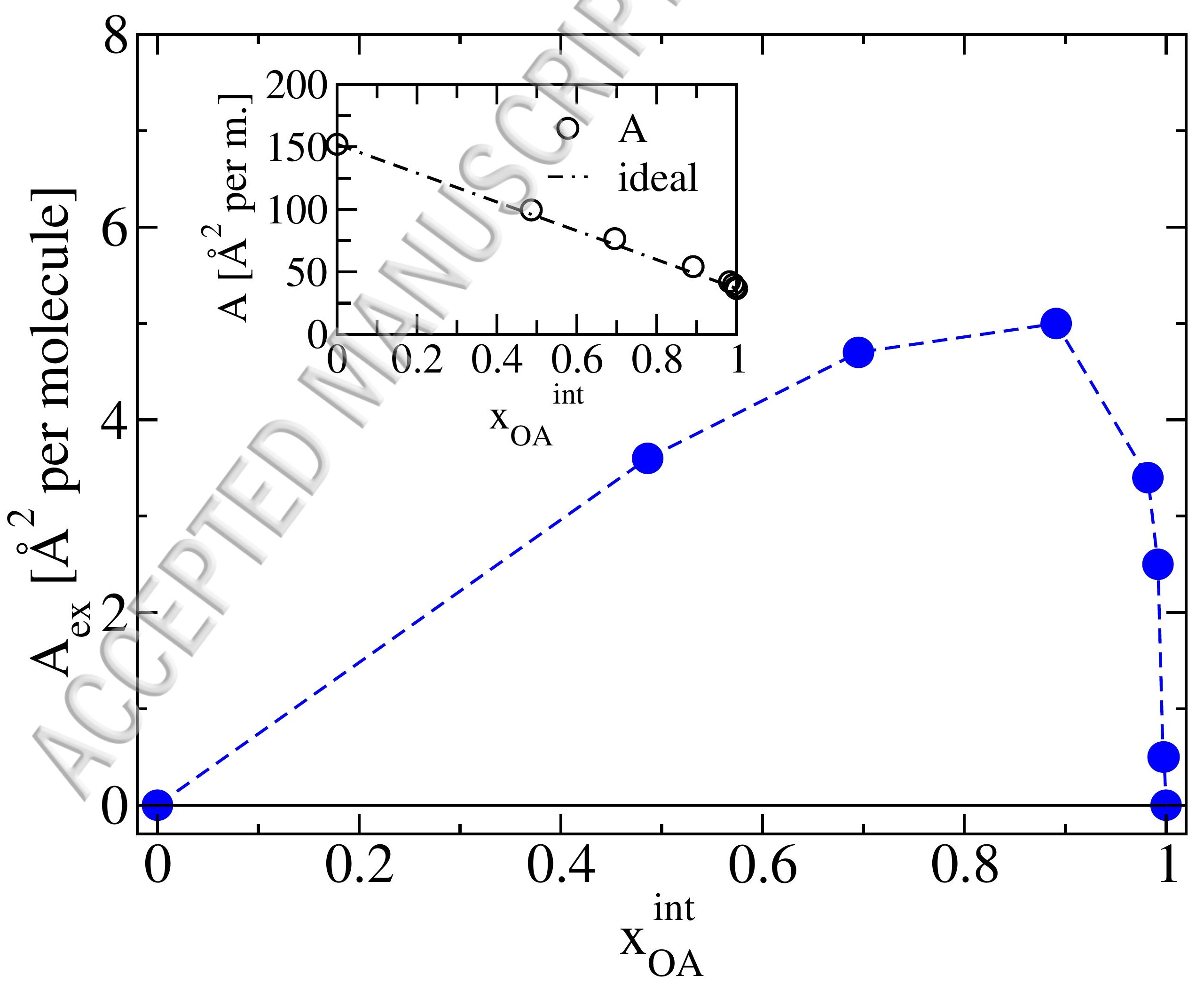

